\title{
Phase Diagrams of Ferromagnet-Superconductor Multilayers with Misaligned Exchange Fields
}

\author{
Tomas Löfwander, Thierry Champel, and Matthias Eschrig \\ Institut für Theoretische Festkörperphysik, Universität Karlsruhe, D-76128 Karlsruhe, Germany
}

(Dated: February 6, 2008)

\begin{abstract}
We study the influence of misalignment of the ferromagnetic exchange field on the equilibrium properties of hybrid structures, composed of superconducting $(\mathrm{S})$ and ferromagnetic $(\mathrm{F})$ parts. In particular, we study numerically the superconducting critical temperature $T_{c}$ in F-S-F trilayers and in F-S-F-S-F Josephson junctions as a function of the misalignment angle $\theta$ of the ferromagnetic magnetization. We discuss the corresponding phase diagrams for these hybrid structures. For the Josephson junctions, a transition between the zero-phase and the $\pi$-phase ground state as a function of $\theta$ takes place under certain conditions. Within the quasiclassical Green's function technique in the diffusive limit, we introduce a fast and effective method for calculating $T_{c}$ in such multilayer structures.
\end{abstract}

\section{INTRODUCTION}

The interest in superconductor-ferromagnet (S-F) hybrid structures has considerably increased in the last decade due to their relevance for the development of nanometer scale electronic devices. The understanding of the superconducting proximity effect in S-F devices is of vital importance for such a goal. Consequently, experimental and theoretical studies have focused on the influence that proximity induced spin-triplet pairing amplitudes in S-F hybrid structures have on superconducting properties of the entire structure. Among those are for example changes of the superconducting transition temperature $T_{c}$ of the device, or the switching between 0 -junctions and $\pi$-junctions as ground states in S-F-S Josephson devices as a function of some control parameter.

The superconducting critical temperature $T_{c}$ in diffusive hybrid $\mathrm{S}-\mathrm{F}$ structures has been studied both theoretically $1,2,3,4,5,6,7,8,9,10,11$ and experimentally $12,13,14,15,16,17,18,19,20,21$ in several recent publications. It has been shown 1.2 that $T_{c}$ has a non-monotonic dependence on the thickness $d_{f}$ of the ferromagnetic layers that provide information about the strength of the ferromagnetic exchange field and about the transparencies of the S-F interfaces. Approximate analytic formulas for $T_{c}$ have been derived for several limiting cases, 23 e.g. for thin or thick film thicknesses or for low or high interface resistances. Recently, Fominov et al. developed a numerical method to compute $T_{c}$ for diffusive $\mathrm{S}-\mathrm{F}$ bilayers 8 and symmetric F-S-F trilayers 10 for arbitrary model parameters such as layer thicknesses and interface resistances. Such an approach is valuable when theory and experiments are compared in detail with the aim to extract parameters as e.g. the ferromagnetic exchange field or the boundary transparencies.

The possibility ${ }^{1,23,24}$ of a $\pi$-state (characterized by a stable phase difference of $\pi$ between the superconducting order parameters) is now well established experimentally in S-F hybrid structures involving several superconduct- ing layers. Transitions between the 0 and $\pi$ states have been revealed in S-F-S junctions by the oscillations of the critical current when varying the temperature $26,27.28$ or the ferromagnetic thickness. $29,30.31$ The transitions from the 0 to $\pi$ state may also be revealed ${ }^{2}$ by the presence of cusps in the dependence of $T_{c}$ on $d_{f}$. Because the cusps may be confounded with the oscillations of $T_{c}\left(d_{f}\right)$ themselves, such a feature in the dependence of $T_{c}$ has been identified experimentally only recently. ${ }^{17.32}$

The presence of several ferromagnetic layers introduces a new degree of freedom, the relative orientation angle, $\theta$, between the magnetizations. The influence of the orientation on $T_{c}$ has been first studied theoretically in $\mathrm{F}$ S-F trilayers in the Refs. 5 and 6 (these authors only considered parallel or antiparallel orientations). The calculations for an arbitrary orientation were performed in Ref. 10. A dependence of the critical current oscillations on the magnetization orientation has been also established theoretically in S-F-F'-S junctions $33,34,35,36$ and multilayered S-F junctions ${ }^{37}$ In Ref. 34 and 37 a switch between the 0 and $\pi$ states has been found from calculations of the Josephson critical current by changing the mutual orientation between the moments. The dependence of $T_{c}$ on the moment orientation (parallel or antiparallel) of trilayers has been studied experimentally in Refs. 16 19 20 21. A dependence on the domain state of the ferromagnet in a S-F bilayer was found in Ref. 22 .

Motivated by the recent experimental studies, we have developed a fast and effective method that is particularly suited for the numerical calculation of $T_{c}$ in diffusive hybrid structures. An important part of this paper is to present details of this method and discuss the calculations leading to some of the results presented in Ref. 32 . Our method can be considered as a development of the method of Fominov et al., who in Refs 8 have presented calculations of $T_{c}$ of $\mathrm{S}-\mathrm{F}$ bilayers and symmetric F-S-F trilayers with non-collinear magnetizations. We extend the calculations to the more general case of asymmetric trilayers in connection with the geometry considered typically in experiments. Within our model, we also treat symmetric pentalayers, including the possibility of a phase difference $\pi$ between the two superconductors. 
This structure was recently studied experimentally in Ref. 32. From our $T_{c}$ calculations, we predict a switching between 0 - and $\pi$-junctions by the orientation of the ferromagnetic exchange fields in pentalayers consisting of a central Josephson junction, two superconductors separated by a ferromagnet, sandwiched between two outer ferromagnets with exchange fields rotated relative to the central ferromagnetic layer. This kind of structure could be realized e.g. by fixing the moments of the outer layers, while rotating the moment of the central layer with an external magnetic field.

The outline of the paper is as follows. In Section \we present the model of the F-S-F trilayer and the F-S-F-S$\mathrm{F}$ pentalayer structures and outline the method that we use to compute the order parameter profile and $T_{c}$ of the structures. In Sections III and IV we present the results for the trilayer and pentalayer respectively. In Section $\nabla$ we discuss some details of our numerical method. We summarize our work in Section DI Some of the technical details have been collected in the appendices.

\section{MODEL AND METHOD}

We shall restrict our considerations to diffusive hybrid structures and to temperatures $T$ near the critical temperature $T_{c}$. We employ a Green's function method in the quasiclassical approximation. The central quantity in this framework is the $2 \times 2$ spin-matrix anomalous Green's function $f$, describing superconducting correlations in the structure. The spin degree of freedom has to be kept due to the fact that the ferromagnets in proximity with the superconductors break spin rotational invariance. Thus, both spin singlet and spin triplet proximity pair amplitudes are present in the ferromagnet. We use a notation where the spin structure is described as $f=\left(f_{s}+\boldsymbol{\sigma} \cdot \mathbf{f}_{t}\right) i \sigma_{y}$, where $\boldsymbol{\sigma}=\left(\sigma_{x}, \sigma_{y}, \sigma_{z}\right)$ are the three Pauli matrices. The pair amplitudes are the spin singlet component $f_{s}$ and the three spin triplet components described by the vector $\mathbf{f}_{t}$. The ferromagnetic regions are characterized by an exchange field with a fixed direction. In the case of rapid changes on the scale of the coherence length of the direction of the exchange field, 38.39 .40 or spin-active interface scattering, $\stackrel{41}{\Perp}$ long-range equal-spin triplet correlations are also induced. We refer to our recent papers 41.42 .43 .44 and a recent review $\underline{45}$ and references therein for a deeper discussion of the origin of these correlations.

For diffusive structures the Green's function is isotropic to lowest order in $1 / p_{f} \ell$, where $p_{f}$ is the Fermi momentum and $\ell$ is the mean free path. Furthermore, for temperatures near the critical temperature the superconducting gap is small $\Delta \ll T_{c}$ and the usual Green's function is approximately equal to the normal state Green's function $g \approx-i \pi \operatorname{sgn}\left(\varepsilon_{n}\right)$, while the anomalous Green's function $f$ is small, of the order of $\Delta$. The relevant starting point in this case is Usadel's diffusion equation ${ }^{46}$ linearized for small $\Delta$. We assume for simplicity that the spatial dependence in the structure is only along the interface normal, taken to be along the $x$-axis, see Figs. 1 and 8 Then, the linearized Usadel equations have the form 43

$$
\begin{aligned}
\left(D \partial_{x x}^{2}-2\left|\varepsilon_{n}\right|\right) f_{s} & =-2 \pi \Delta+2 i \operatorname{sgn}\left(\varepsilon_{n}\right) \mathbf{J} \cdot \mathbf{f}_{t} \\
\left(D \partial_{x x}^{2}-2\left|\varepsilon_{n}\right|\right) \mathbf{f}_{t} & =2 i \operatorname{sgn}\left(\varepsilon_{n}\right) \mathbf{J} f_{s}
\end{aligned}
$$

where $\operatorname{sgn}\left(\varepsilon_{n}\right)$ is the sign of the Matsubara frequency $\varepsilon_{n}=\pi T(2 n+1)$, and we have used the short hand notation $f=f\left(\varepsilon_{n}, x\right)$. We assume that the exchange field $\mathbf{J}=\mathbf{J}(x)$ is non-zero in the ferromagnetic regions, while $\Delta=\Delta(x)$ is non-zero in the superconducting regions. Each layer in the structure can have a different diffusion constant $D$. Note that we assume that the exchange field is small compared to the Fermi energy, $J \ll \epsilon_{f}$, in which case the quasiclassical theory can be straightforwardly applied. For strong exchange fields, a separate calculation has to be made 41

The diffusion equation is supplemented with boundary conditions at each interface and at the outer surfaces of the structure. The boundary condition connecting the Green's function at $x_{S}$ on the superconductor side of the interface with the Green's function at $x_{F}$ on the ferromagnet side of the interface is of the form first derived by Kupriyanov and Lukichev $\underline{47}$

$$
\begin{aligned}
\gamma \xi_{F} f^{\prime}\left(x_{F}\right) & =\xi_{S} f^{\prime}\left(x_{S}\right), \\
\gamma_{b} \xi_{F} f^{\prime}\left(x_{F}\right) & = \pm\left[f\left(x_{S}\right)-f\left(x_{F}\right)\right],
\end{aligned}
$$

where $\xi=\sqrt{D / 2 \pi T_{c 0}}$ is the coherence length and the parameters $\gamma$ and $\gamma_{b}$ characterize the conductivity mismatch between the two sides and the boundary resistance, respectively. The sign in Eq. (4) is positive (negative) for a $\mathrm{F} / \mathrm{S}(\mathrm{S} / \mathrm{F})$ interface [for which the superconductor occupies the space to the right (left) of the barrier]. Note that we use the prime as a short-hand notation for spatial derivatives at a certain point in space, e.g. $f^{\prime}\left(x_{S}\right)=\left.\partial_{x} f(x)\right|_{x=x_{S}}$. At the outer surfaces of the structure, we require that the current trough the boundary must vanish, i.e. $\partial_{x} f=0$.

Since the exchange field and the superconducting order parameter are spatially separated, we see that in the superconducting region Eqs. (12)-(21) are decoupled. The triplet part (2) can be solved analytically, while the singlet part (11) has a source term containing the order parameter that satisfies the self-consistency equation

$$
\Delta(x) \ln \frac{T}{T_{c 0}}=T \sum_{\varepsilon_{n}}\left(f_{s}\left(\varepsilon_{n}, x\right)-\frac{\pi \Delta(x)}{\left|\varepsilon_{n}\right|}\right) .
$$

In the ferromagnetic regions Eqs. (11)-(2) are coupled but the superconducting order parameter is absent and both equations can be solved analytically, which is described in detail in Appendix $\mathbb{A}$ and $\mathbb{B}$ for the trilayer and pentalayer cases. The presence of the ferromagnetic regions are in the process reduced to an effective boundary condition for the calculation of the singlet component in the superconducting region, which we confine to 
$0<x<d_{s}$ in the present discussion, as in Fig. 1 The boundary condition can in the general case be written in the form

$$
\left(\begin{array}{c}
f_{s}^{\prime}(0) \\
f_{s}^{\prime}\left(d_{s}\right)
\end{array}\right)=k_{s} \hat{W}\left(\begin{array}{c}
f_{s}(0) \\
f_{s}\left(d_{s}\right)
\end{array}\right)
$$

where $k_{s}=\sqrt{2 \varepsilon_{n} / D_{s}}$ and $\hat{W}$ is a $2 \times 2$ matrix. The non-locality of the boundary condition (6), i.e. the coupling of the two interfaces at 0 and at $d_{s}$, is a result of the coupling of the singlet and triplet anomalous Green's functions $f_{s}$ and $\mathbf{f}_{t}$ in the original boundary conditions Eqs. (3)-(4) and the coupling of the diffusion equations for the singlet and triplet components in the ferromagnet by the exchange field. The matrix $\hat{W}$ depends on the Matsubara frequency, the parameters of the adjacent layers (thicknesses, exchange fields, diffusion constants), and the interface parameters $\gamma$ and $\gamma_{b}$. The expressions for the components of $\hat{W}$ are derived in Appendix $\mathrm{A}$ and B for the trilayer and pentalayer structures. The following method for calculating $T_{c}$ is however applicable for any matrix $\hat{W}$, as long as the boundary condition for the singlet Green's function $f_{s}$ is of the form (6).

Consider Eq. (1) in the superconducting region, i.e. for $0<x<d_{s}$ where $\mathbf{J}=0$. By linear superposition we have ${ }^{8}$

$$
f_{s}\left(\varepsilon_{n}, x\right)=\pi \int_{0}^{d_{s}} G\left(\varepsilon_{n}, x, y\right) \Delta(y) d y,
$$

where the function $G\left(\varepsilon_{n}, x, y\right)$ is the solution of the differential equation

$$
\left(\frac{D_{s}}{2} \partial_{x x}^{2}-\left|\varepsilon_{n}\right|\right) G\left(\varepsilon_{n}, x, y\right)=-\delta(x-y),
$$

subject to the boundary conditions ([6) with $f_{s}\left(\varepsilon_{n}, x\right)$ replaced by $G\left(\varepsilon_{n}, x, y\right)$. The solution of Eq. (8) is presented in Appendix W With the help of the function $G$, the gap equation can be written as

$$
\frac{2 \pi T \sum_{\varepsilon_{n}>0} \int_{0}^{d_{s}} G\left(\varepsilon_{n}, x, y\right) \Delta(y) d y}{\ln \frac{T}{T_{c} 0}+2 \pi T \sum_{\varepsilon_{n}>0}\left(\varepsilon_{n}\right)^{-1}}=\Delta(x),
$$

where we used that the singlet Green's function $f_{s}\left(\varepsilon_{n}, x\right)$ [and therefore also $G\left(\varepsilon_{n}, x, y\right)$ ] is an even function of $\varepsilon_{n}$. We see that one way ${ }^{8}$ of solving the problem at hand is to discretize the spatial coordinate $\left(x \rightarrow x_{k}, k=1 \ldots N\right)$ and find the critical temperature $T_{c}$ as the highest temperature for which the eigenvalue of the $N \times N$ matrix on the left hand side of Eq. (9) equals one. The corresponding eigenvector gives the profile of the order parameter, $\Delta\left(x_{k}\right)$.

There are several disadvantages of the method described above, all connected with the discretization of the spatial coordinate axis. In particular, it is cumbersome to reach acceptable numerical accuracy when $T_{c}$ is computed. We shall discuss these problems in detail in Section $\nabla$
Because of these draw backs, we develop a Fourier series method that avoids the discretization of the spatial coordinate. The superconducting order parameter $\Delta(x)$ exists in the range $0<x<d_{s}$. We extend its domain of definition to the full real axis by adding an even-parity property and $2 d_{s}$ periodicity. Then, $\Delta(x)$ can be expanded in a Fourier series

$$
\Delta(x)=\sum_{p=0}^{\infty} \Delta_{p} \cos \left(\frac{p \pi x}{d_{s}}\right),
$$

where the coefficients $\Delta_{p}$ are defined as

$$
\Delta_{p}=\frac{2-\delta_{p 0}}{d_{s}} \int_{0}^{d_{s}} \Delta(x) \cos \left(\frac{p \pi x}{d_{s}}\right) d x .
$$

We show in Appendix $\mathbb{D}$ how to obtain an analytic expression for the singlet amplitude $f_{s}$ in terms of the Fourier coefficients $\Delta_{p}$. Consequently, the gap equation can be written in the space of Fourier coefficients as

$$
\sum_{p=0}^{\infty} m_{l p} \Delta_{p}=0
$$

for integer $l \geq 0$, and where $m_{l p}$ are given in Eqs. (D3)(D4). We solve the problem at hand by introducing a cutoff $p_{c}$ for the number of harmonics and find the critical temperature $T_{c}$ as the highest temperature for which the eigenvalue of the $p_{c} \times p_{c}$ matrix on the left hand side of Eq. (12) equals zero. The corresponding eigenvector gives the profile of the order parameter $\Delta(x)$ through the sum in Eq. (10).

In the following two section we use this method to compute $T_{c}$ for the S-F trilayer and pentalayer structures. In Section $\nabla$ we discuss the advantages of our method [Eq. (12)] and compare with the other method [Eq. (9)].

\section{TRILAYER}

Consider the trilayer structure shown in Fig. [ We study in this section the superconducting transition temperature of such a trilayer. Our studies are motivated by the recent experiments on S-F layered structures, 12.13 .14 .15 .16 .17 .18 .19 .20 .21 including in particular the experiments in Ref. 32 on the critical temperature of asymmetric $\mathrm{F}_{1}-\mathrm{S}-\mathrm{F}_{2}$ trilayers. The theory fits of $T_{c}$ of the trilayers in Ref. 32 were obtained with the theory presented in the present paper.

In the left ferromagnetic layer $\left(\mathrm{F}_{1}\right)$, the exchange field is aligned with the $z$-axis, while in $\mathrm{F}_{2}$ it lies in the $y z$ plane and forms an angle $\theta$ with respect to the $z$-axis. The origin of the coordinate system is taken at the $\mathrm{F}_{1} / \mathrm{S}$ interface. The two layers $\mathrm{F}_{1}$ and $\mathrm{F}_{2}$ are characterized by their thicknesses $\left(d_{f 1}, d_{f 2}\right)$, exchange fields $\left(J_{1}, J_{2}\right)$ and diffusion constants $\left(D_{f 1}, D_{f 2}\right)$, while the superconducting layer is characterized by its thickness $\left(d_{s}\right)$, pairing interaction strength (i.e. the bulk material superconducting critical temperature $T_{c 0}$ ), and diffusion constant $\left(D_{s}\right)$. The diffusion constants are converted into 


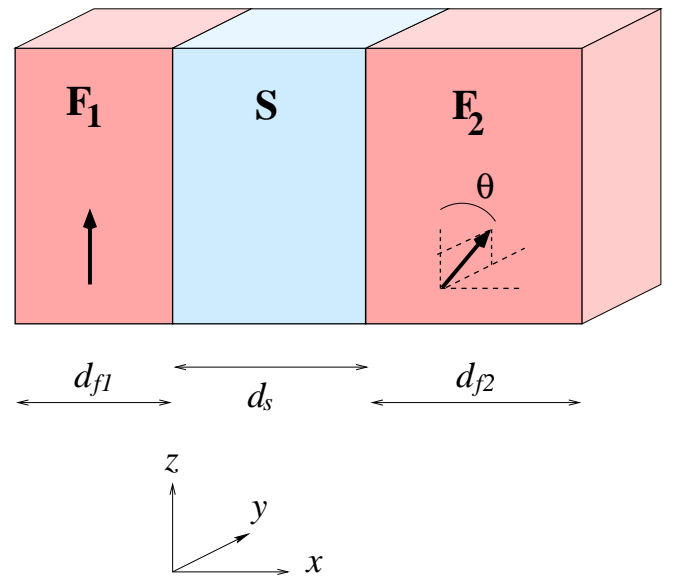

FIG. 1: Geometry of the asymmetric $\mathrm{F}_{1}-\mathrm{S}-\mathrm{F}_{2}$ structure. The moments $\mathbf{J}_{1}$ (in $\mathrm{F}_{1}$ ) and $\mathbf{J}_{2}$ (in $\mathrm{F}_{2}$ ) may have different amplitudes and point in different directions (the relative orientation angle is denoted $\theta$ ).

coherence lengths $\xi=\sqrt{D / 2 \pi T_{c 0}}$ and we shall use the coherence length in the superconductor $\xi_{s}$ as length scale in the problem. The $\mathrm{F}_{1} / \mathrm{S}$ and $\mathrm{S} / \mathrm{F}_{2}$ interfaces are characterized by the conductivity mismatches $\left(\gamma_{1}, \gamma_{2}\right)$ and interface resistances $\left(\gamma_{b 1}, \gamma_{b 2}\right)$.

The Usadel equations (11)-(21) are solved as described in Appendix $\mathrm{A}$ to give the effective boundary condition matrix $\hat{W}$ for the trilayer. The matrix $m_{l p}$ of Eq. (12) is then given in terms of the elements of $\hat{W}$ as shown in Appendix D.

\section{A. Results}

In Fig. 23 we present the influence of an exchange field on $T_{c}$ for an asymmetric $\mathrm{F}_{1}-\mathrm{S}-\mathrm{F}_{2}$ trilayer (with $d_{f 1} \neq d_{f 2}$ ). In the normal metal case (obtained by setting $J=0$ ), the critical temperature is monotonically suppressed as the layer thickness $d_{f 1}$ is increased, see Fig. 2 In the case of a ferromagnet, the exchange field induces an additional oscillatory behavior, closely connected to the spin mixing between up and down spins. As a result, $T_{c}$ is suppressed in a non-monotonic way. For a strong enough exchange field, the oscillation is so strong that superconductivity is suppressed at a critical thickness but can reappear at a larger thickness. This kind of non-monotonic dependence of $T_{c}$ was thoroughly studied $4,5,6.8,10,11,23$ for F-S bilayers and symmetric F-S$\mathrm{F}$ trilayers. The crossing of the two $T_{c}$ curves in Fig. [3] is due to the non-monotonic $d_{f 1}$ dependence shown in Fig. [2]

The exact point where superconductivity disappears depends on many other parameters in addition to the strength of the exchange field. For example, the influence of the second ferromagnet's thickness $d_{f 2}$ can be understood in terms of the initial $T_{c}$ suppression at $d_{f 1}=0$, which corresponds to the bilayer case. For sufficiently

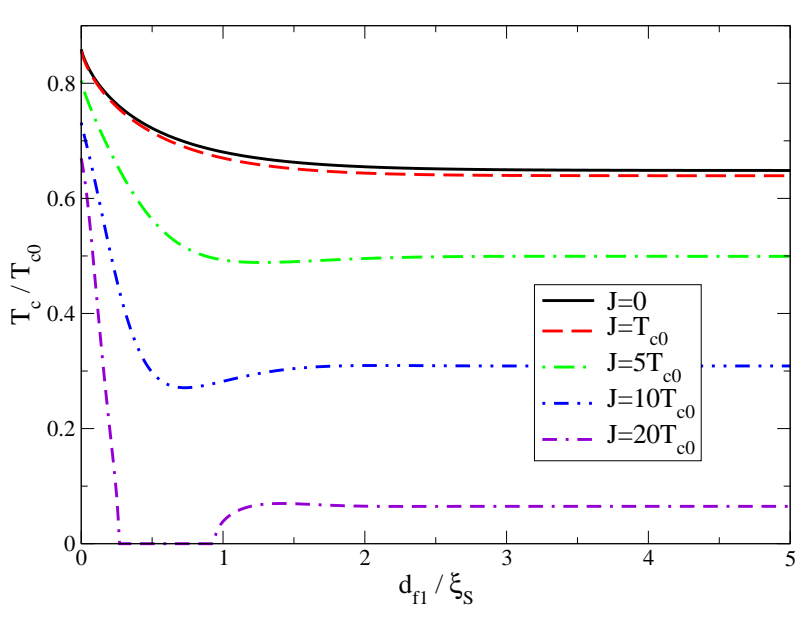

FIG. 2: Critical temperature $T_{c}$ of a trilayer versus the thickness of the left ferromagnet for several strengths of the exchange field ranging from $J_{1}=J_{2}=J=0$ (the normal metal case) to strong $J=20 T_{c 0}$. The other layer thicknesses are $d_{s}=2 \xi_{S}$ and $d_{f 2}=0.5 \xi_{S}$, the interface parameters are $\gamma_{1}=\gamma_{2}=0.3$ and $\gamma_{b 1}=\gamma_{b 2}=0.7$, the exchange fields are parallel $(\theta=0)$, and the diffusion constants are equal, $D_{f 1}=D_{f 2}=D_{S}$.

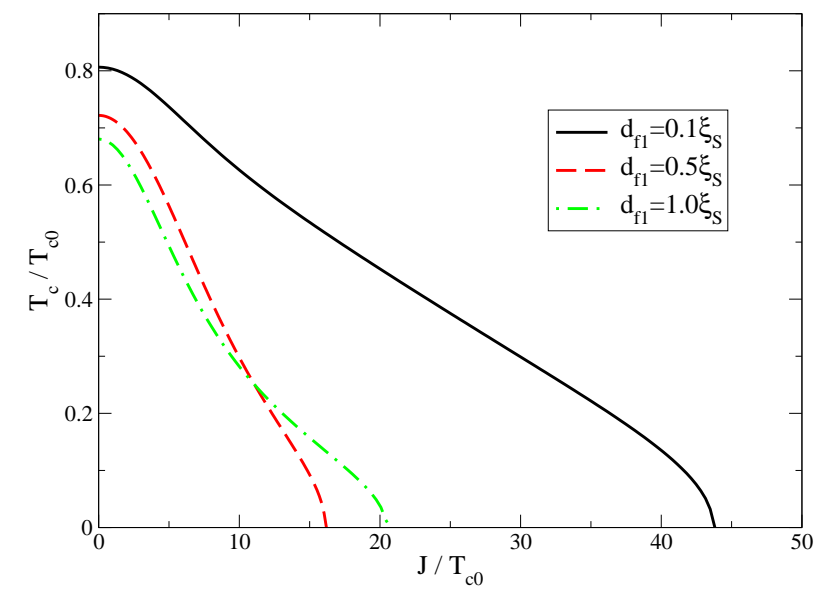

FIG. 3: Critical temperature $T_{c}$ of a trilayer versus the strength of the exchange fields $J_{1}=J_{2}=J$ for a few layer thicknesses $d_{f 1}$ of one of the ferromagnetic layers. The other parameters are the same as in Fig. 2

large $d_{f 2}$ the initial suppression is large enough that the subsequent oscillations with increasing $d_{f 1}$ lead to disappearing and reappearing superconductivity, see Fig. [4 Naturally, the initial $T_{c}$ suppression at $d_{f 1}=0$ is a nonmonotonic function of $d_{f 2}$, in analogy to the $T_{c}\left(d_{f 1}\right)$ dependence.

Another important parameter for the size of the $T_{c}$ variations, is the interface resistance. As seen in Fig. [5 superconductivity is suppressed in trilayers with good contacts (small $\gamma_{b}$ ) for the model parameters chosen here. For example it is not possible to consider $d_{s} \ll \xi_{s}$, for which simplified calculations with a constant $\Delta$ can be made, and simultaneously consider good contacts $\gamma_{b} \rightarrow 0$ 


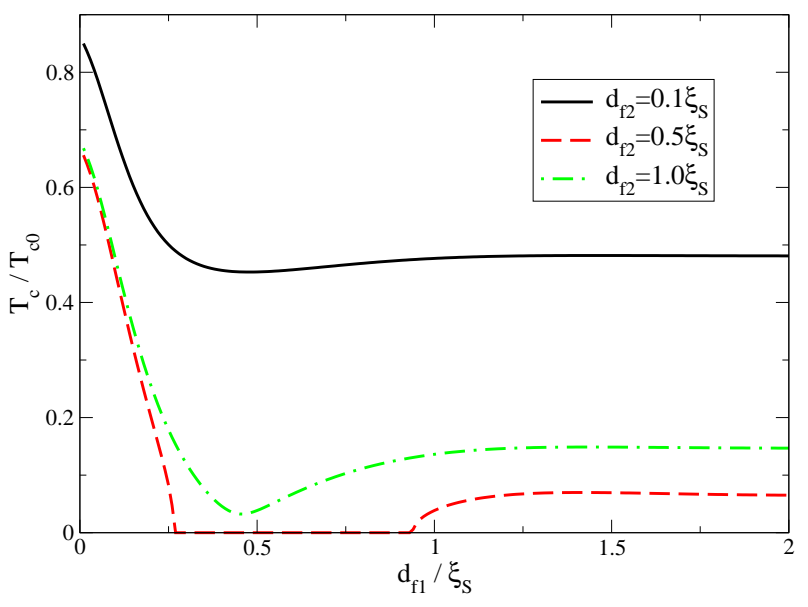

FIG. 4: Critical temperature $T_{c}$ of a trilayer versus the thickness $d_{f 1}$ of the left ferromagnet for several thicknesses $d_{f 2}$ of the right ferromagnet. The exchange field is $J_{1}=J_{2}=20 T_{c 0}$ and the other parameters are the same as in Fig. 2

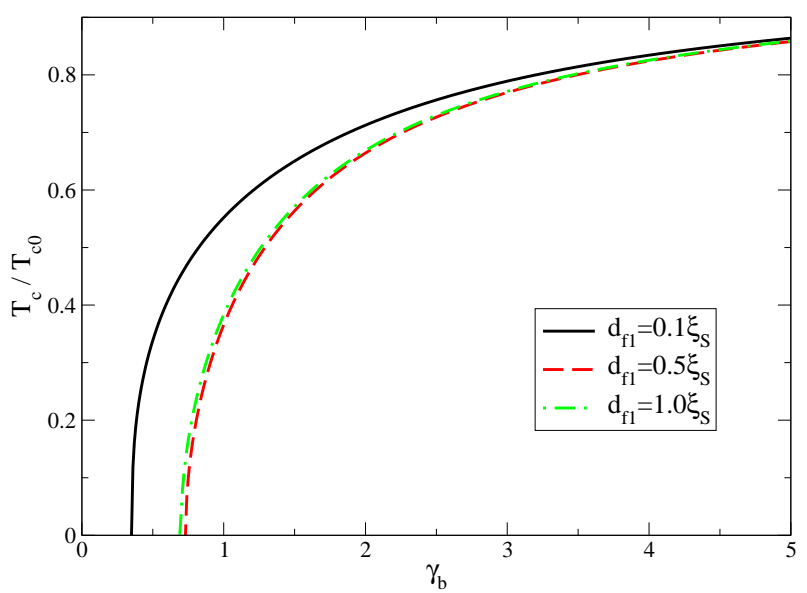

FIG. 5: Critical temperature $T_{c}$ of a trilayer versus the interface resistance parameter $\gamma_{b}=\gamma_{b 1}=\gamma_{b 2}$ at a few thicknesses $d_{f 1}$ corresponding to points on the $J=20 T_{c 0}$ curve in Fig. [2 to the left, inside, and to the right of the $T_{c}=0$ region. The other parameters are the same as in Fig. 2

for reasonable conductivity mismatches (here $\gamma=0.3$ ). Certainly, for larger conductivity mismatches (smaller $\gamma$ ), $T_{c}$ is not suppressed as much and a smaller $\gamma_{b}$ can be used. However, it is always important to keep in mind that $T_{c}$ is suppressed to zero in quite a large parameter space, including small $d_{s}$ and small $\gamma_{b}$.

In Fig. [6] we show the influence of the relative direction of the exchange fields in the two ferromagnetic layers. The dependence is monotonic, with the parallel orientation being the most destructive. We note (see also Ref. 10) that for parallel or antiparallel exchange field orientations triplet correlations with zero spin projection on the local exchange field are present in the structure, while for intermediate orientations triplet correlations with non-zero projection are also induced. In order to describe the $\theta$-dependence correctly, it is therefore im-

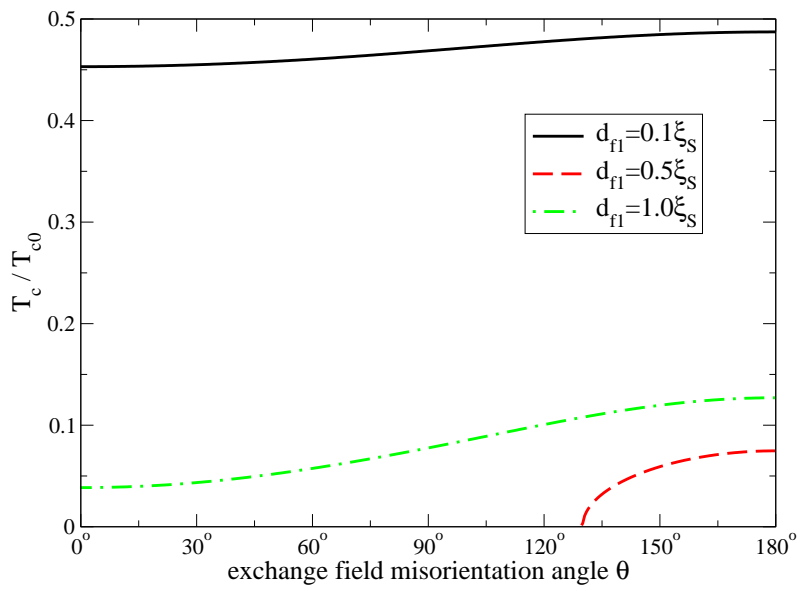

FIG. 6: Critical temperature $T_{c}$ of a trilayer versus the misorientation angle $\theta$ between the exchange fields in ferromagnets $\mathrm{F}_{1}$ and $\mathrm{F}_{2}$. The exchange field is $J_{1}=J_{2}=20 T_{c 0}$ and the other parameters are the same as in Fig. 2
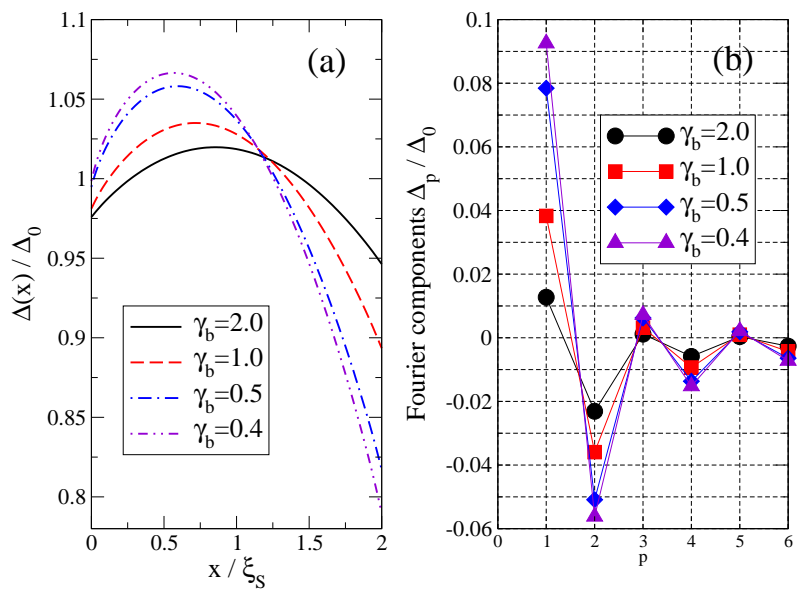

FIG. 7: (a) The spatial dependence of the order parameter for several interface resistance parameters $\gamma_{b}=\gamma_{b 1}=\gamma_{b 2}$ on the solid curve $\left(d_{f 1}=0.1 \xi_{S}\right)$ in Fig. 5 (b) The Fourier components in Eq. (10). Note that $\Delta$ is normalized to the first component $\Delta_{0}$, which remains unknown in a linearized theory.

portant to include $\vec{f}_{t}$, see Appendix $\AA$

In Fig. 7 a) we present order parameter profiles for four different values of $\gamma_{b}$ on the solid line $\left(d_{f 1}=0.1 \xi_{S}\right)$ in Fig. [5 For a good contact (small resistance $\gamma_{b}$ ), the pair breaking becomes quite severe. The suppression is reflected as a growth of the Fourier components $p \geq 1$, see Fig. Z(b).

\section{PENTALAYER}

Consider the pentalayer shown in Fig. 8 Experimental results for the critical temperature, including signatures of a transition from a zero- to a $\pi$-junction as function of the thickness of the central F-layer, were recently pre- 


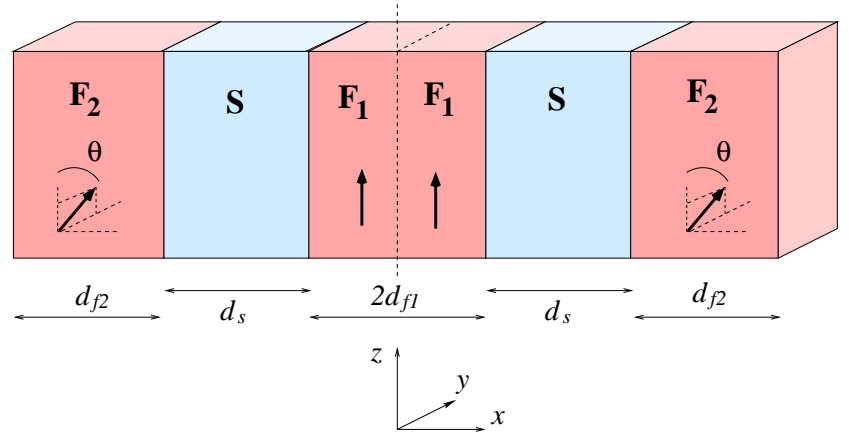

FIG. 8: The $\mathrm{F}_{2} / \mathrm{S} / \mathrm{F}_{1} / \mathrm{S} / \mathrm{F}_{2}$ pentalayer structure. We consider two types of misalignment of the outer exchange fields relative to the exchange field in the center layer. First, as shown here, $J_{2}$ is rotated by the same angle $\theta$. The second possibility is when $J_{2}$ is rotated in opposite directions, $-\theta$ in the left $\mathrm{F}_{2}$ and $+\theta$ in the right $\mathrm{F}_{2}$.

sented for this structure in Ref. 32. The theory fits of $T_{c}$ of the pentalayers in Ref. 32 were obtained with the theory presented in the present paper.

The superconducting layers are considered geometrically identical with identical bulk material critical temperatures $T_{c 0}$. In the central ferromagnetic layer $\left(\mathrm{F}_{1}\right)$, the exchange field is aligned with the $z$-axis, while in the right and left layers $\left(\mathrm{F}_{2}\right)$ it forms an angle $\theta$ with respect to the $z$-axis. We characterize the different layers by their thicknesses, exchange fields, and diffusion constants, with the constraint that the pentalayer should have certain symmetries with respect to the midpoint, see below. The present pentalayer problem can then be reduced to a trilayer problem with a new effective boundary condition at a fictitious outer surface at the center $(x=0)$. The two superconducting order parameters in the left and right $\mathrm{S}$ layers may differ in phase, which is reflected in the effective boundary condition.

We shall consider two types of misorientation of the exchange fields in the outer layers relative to the center layer: the exchange fields $J_{2}$ are rotated by $+\theta$ as in Fig. 8 (rotation type $1,+\theta /+\theta$ ), or rotations by $-\theta$ and $+\theta$ in the left and right outer layers respectively (rotation type $2,-\theta /+\theta)$.

For rotation type $1(+\theta /+\theta)$, when the phase difference vanishes (0-junction case), the singlet component $f_{s}$ is an even function of $x$. Considering the parity of the exchange field $\mathbf{J}\left(J_{z} \rightarrow J_{z}\right.$ and $\left.J_{y} \rightarrow J_{y}\right)$ and the Eqs. (11)-(2), we deduce that the $f_{t z}$ and $f_{t y}$ components have the same even parity. Thus, we impose the conditions

$$
(+\theta,+\theta), 0-\text { jct }: f_{s}^{\prime}(0)=f_{t z}^{\prime}(0)=f_{t y}^{\prime}(0)=0 .
$$

On the other hand, when the phase difference is $\pi(\pi$ junction case), $f_{s}, f_{t z}$ and $f_{t y}$ are odd functions of $x$ and we impose the conditions

$$
(+\theta,+\theta), \pi-\text { jct }: f_{s}(0)=f_{t z}(0)=f_{t y}(0)=0 .
$$

For rotation type $2(-\theta /+\theta)$, the exchange field component $J_{y}$ is instead odd under $x \rightarrow-x$. For the 0 -

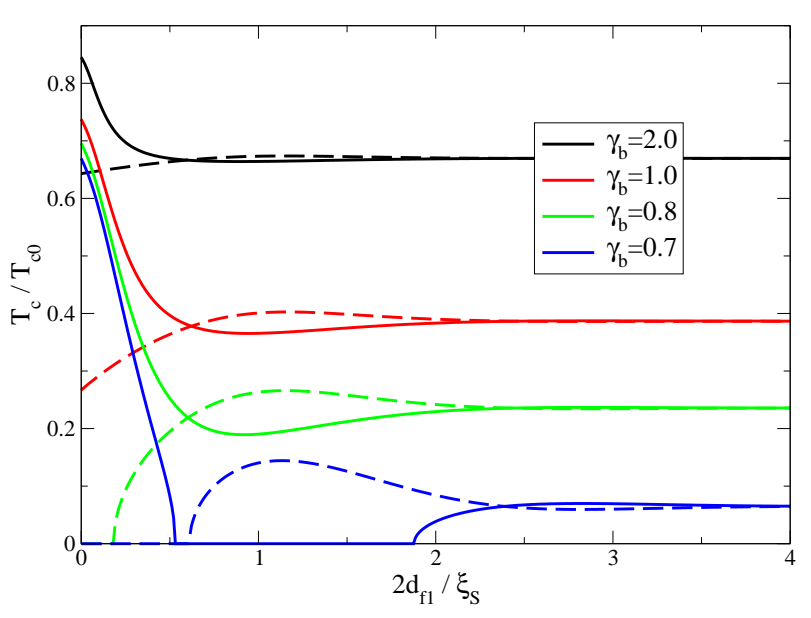

FIG. 9: Critical temperature $T_{c}$ of a pentalayer versus the center ferromagnet layer thickness $2 d_{f 1}$ for several barrier transparencies. The curves come in pairs, solid line for the 0 -junction and dashed line for the $\pi$-junction, from top to bottom for $\gamma_{b 1}=\gamma_{b 2}=\gamma_{b}=\{2,1,0.8,0.7\}$ respectively. The other parameters are $d_{S}=2 \xi_{S}, d_{f 2}=0.5 \xi_{S}, J_{1}=J_{2}=20 T_{c 0}$, $\theta=0, \gamma_{1}=\gamma_{2}=0.3$, and $D_{f 1}=D_{f 2}=D_{S}$.

junction case, it implies that $f_{t y}$ is odd, while the other components are even just as above. For the $\pi$-junction case the parities are interchanged. The effective boundary conditions are

$$
\begin{aligned}
&(-\theta /+\theta), 0-\text { jct }:\left\{\begin{array}{l}
f_{s}^{\prime}(0)=f_{t z}^{\prime}(0)=0, \\
f_{t y}(0)=0,
\end{array}\right. \\
&(-\theta /+\theta), \pi-\text { jct }: \quad\left\{\begin{array}{l}
f_{s}(0)=f_{t z}(0)=0, \\
f_{t y}^{\prime}(0)=0 .
\end{array}\right.
\end{aligned}
$$

As shown in Appendix $[\mathrm{B}$ the different boundary conditions yield different matrices $\hat{W}$ for the effective boundary condition (6).

\section{A. Results}

The dependence of $T_{c}$ on the various parameters in the model is similar for the trilayer with left ferromagnetic layer thickness $d_{f 1}$ and for the pentalayer with a phase difference 0 between the two superconductors and a central ferromagnet layer thickness $2 d_{f 1}$. The critical temperature is in fact equal for rotation type I, since the boundary condition at the center of the pentalayer, Eq. (13), is the same as for the outer surface of the trilayer. Note, however, that the boundary condition for one of the triplets is different for rotation type II, see Eq. (15). The new ingredient in the pentalayer case is the possibility of a phase difference $\pi$ between the superconductors. The $\pi$-state can in simplified terms be understood as being due to the oscillatory behavior of the Green's function $f_{s}\left(\varepsilon_{n}, x\right)$ inside the central ferromagnetic layer $\mathrm{F}_{1}$. In an experiment, $T_{c}$ is given by the largest $T_{c}$ for each thickness and there will be a sudden 

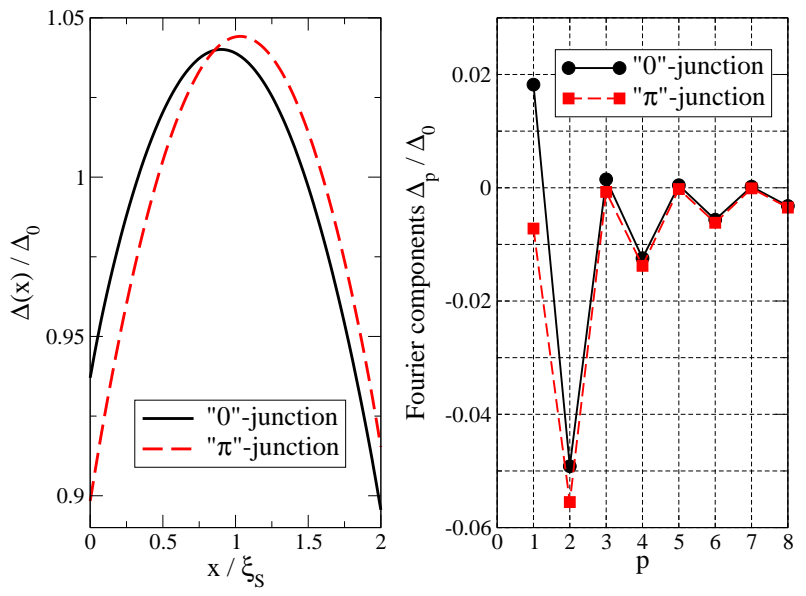

FIG. 10: (a) The order parameter profile for the parameters in Fig. 9 for $\gamma_{b}=0.8$ at $2 d_{f 1}=0.4 \xi_{S}$, for which the critical temperature for the 0 - and $\pi$-junctions are respectively $T_{c} \approx 0.3 T_{c 0}$ and $T_{c}=0.16 T_{c 0}$. (b) The corresponding Fourier components.
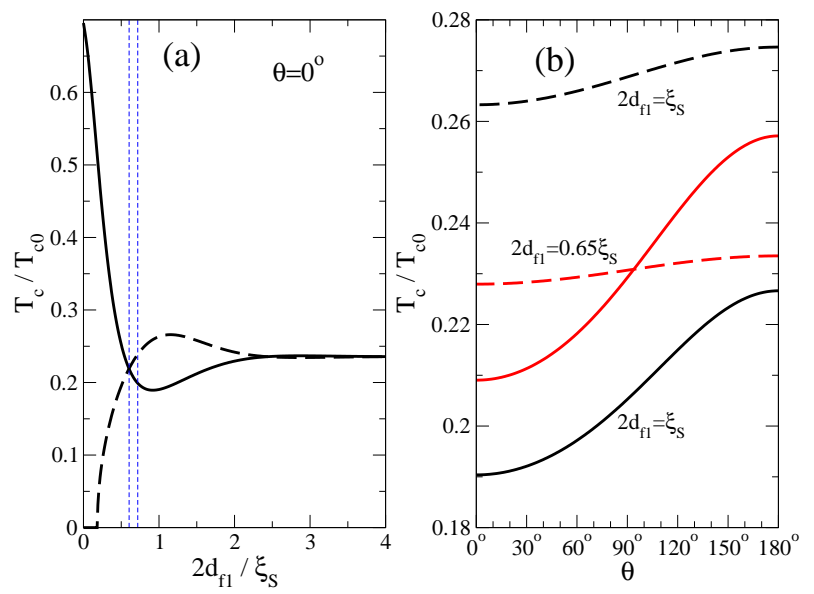

FIG. 11: (a) The same curves as in Fig. 9 for $\gamma_{b}=0.8$. For thicknesses $d_{f 1}$ between the two vertical lines the $0 \rightarrow \pi$ transition can be tuned by the relative orientation of the exchange fields in $\mathrm{F}_{1}$ and $\mathrm{F}_{2}$. (b) The switch $0 \rightarrow \pi$ appears at a critical angle $\theta_{c} \approx 84^{\circ}$ for the thickness $2 d_{f 1}=0.65 \xi_{S}$. For the larger thickness $2 d_{f 1}=\xi_{S}$, outside the window indicated in (a), the largest $T_{c}$ is obtained for the $\pi$-junction.

almost kink-like change in $T_{c}$ at the $0 \rightarrow \pi$ transition. For large oscillations, the transition becomes sharper. This is illustrated by changing the interface resistance $\gamma_{b}$ in Fig. 9 For good contacts and strong exchange fields, superconductivity can be destroyed at some critical thickness and then reappear at a larger thickness, just as in the trilayer case. For the pentalayer, however, the $\pi$-phase can pre-empt the 0 -phase and superconductivity appears earlier compared to the trilayer as $d_{f 1}$ is increased, see the curves for $\gamma_{b}=0.7$ in Fig. 9

An example of the order parameter suppression is shown in Fig. 10 (a), corresponding to $\gamma_{b}=0.8$ and $2 d_{f 1}=0.4 \xi_{S}$ in Fig. 9 The suppression of $\Delta$ at the
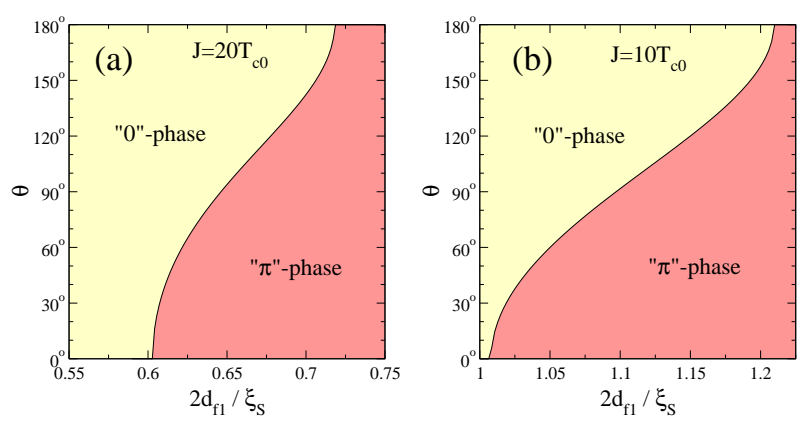

FIG. 12: (a) Phase diagram of the $0 \rightarrow \pi$ transition in the window indicated in Fig. 111 a). In (b) we show the phase diagram for a smaller exchange field, $J=10 T_{c 0}$. The range of thicknesses $d_{f 1}$ for which there is a switching by changing $\theta$ is larger in this case.

interfaces is more severe for phase difference $\pi$ and the 0 -junction is stabilized, i.e. has the largest $T_{c}$ as seen in Fig. 9]

In the region close to the $0 \rightarrow \pi$ transition, it is possible to switch between the 0 - and $\pi$-phases by changing the relative orientation of the exchange fields. We note that this possibility was already deduced from calculations of the Josephson critical current in the papers 34 and 37 considering different geometries. We illustrate this effect in Fig. [11] switching is possible in between the vertical lines in Fig. 111(a). Since experimentally, $T_{c}$ is given by the largest $T_{c}$ for each $\theta$, the $0 \rightarrow \pi$ switch would show up as a sudden almost kink-like change in $T_{c}$ with the variation of $\theta$, as shown in Fig. 111 b). We present in Fig. 12 the phase diagram of the junction in the region around the window indicated in Fig. 11](a). The window inside which a $0 \rightarrow \pi$ phase change can be induced by the orientation angle $\theta$ is larger for a smaller exchange field since the $T_{c}$-oscillation period is longer in this case. We see this effect by comparing the $J=20 T_{c 0}$ case in Fig. 111(a) to the $J=10 T_{c 0}$ case shown in (b).

It has been found ${ }^{8,9,23}$ for the bilayer and trilayer cases that $T_{c}$ can become a multiple valued function of e.g. the thickness of the ferromagnet. We show this type of behavior for the pentalayer case in Fig. 13 (upper panel). The non-monotonic dependence of $T_{c}$ is similar to the case of a clean thin film in an in-plane magnetic field ${ }^{48.49}$ and to thin films of superfluid ${ }^{3} \mathrm{He}$ 50 51]. For these clean systems it has been proposed that an inhomogeneous superconducting state can be formed. In a dirty system, such inhomogeneity seems very unlikely and it has instead been proposed that the back-bend signals the possibility of a first-order transition in the system ${ }^{23}$ First-order transitions are however beyond the scope of the present paper. Instead we point out that for the pentalayer case, the $\pi$-phase becomes favorable in the same region of thicknesses as where there is a back-bend for the 0 -junction. The back-bend behavior for the 0-junction, and the interfering $\pi$-phase, occurs also as function of the exchange field misorientation angle $\theta$, see the lower panel 

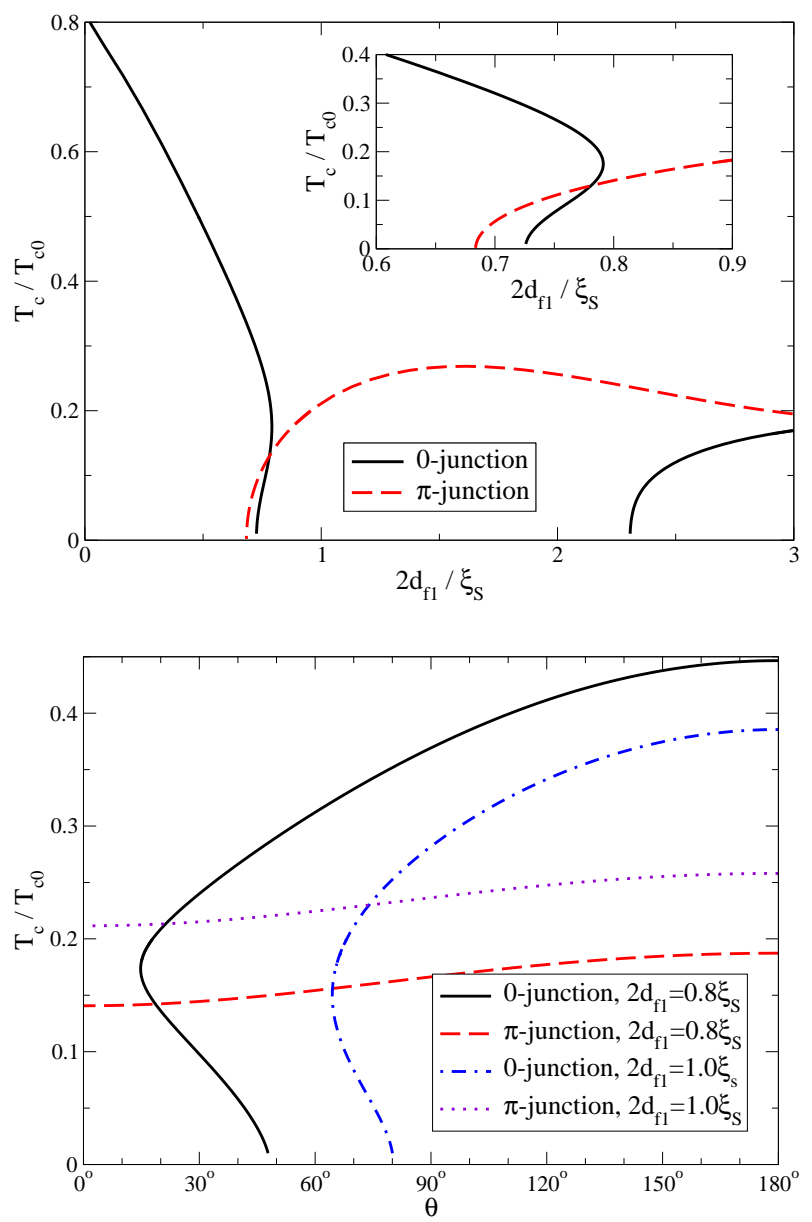

FIG. 13: Upper panel: In the region where $T_{c}$ is suppressed to zero, the curve $T_{c}\left(d_{f 1}\right)$ can contain a back-bend. This latter could signal the occurrence of a first-order transition, which is however beyond the scope of the present theory. For the pentalayer, the $\pi$-phase can interfere and the first order transition might be avoided. The parameters are $d_{s}=2 \xi_{s}$, $d_{f 2}=0.2 \xi_{s}, \gamma_{1}=\gamma_{2}=0.35, \gamma_{b 1}=\gamma_{b 2}=0.4, J_{1}=J_{2}=10 T_{c 0}$ and $\theta=0$. In the lower panel we study the dependence on the exchange field for two particular thicknesses $d_{f 1}$ in the upper panel. Clearly, the back-bend behavior can occur also as function of the exchange field misorientation angle $\theta$.

in Fig. 113 Interestingly, there is a discontinuous drop in $T_{c}$ at the $0 \rightarrow \pi$ transition when $\theta$ is tuned from around $20^{\circ}$ down to $10^{\circ}$, see the solid and dashed lines in Fig. 113

For very large thicknesses $d_{f 1}$ the predominant superconducting correlations that penetrate $\mathrm{F}_{1}$ and connect the two superconductors are the long-range nonoscillatory triplet components of $\mathbf{f}_{t}$. As a consequence, at large $d_{f 1}, T_{c}$ becomes a monotonic function of $d_{f 1}$. The difference in $T_{c}$ between the 0 - and $\pi$-phases is, however, quite small, see Fig. 14. The junction is stabilized at large $d_{f 1}$ either at 0 or at $\pi$ phase difference, depending on the way the exchange fields of the two outer ferromagnetic layers are rotated relative to the center layer (a similar effect associated with the chirality of the rotation
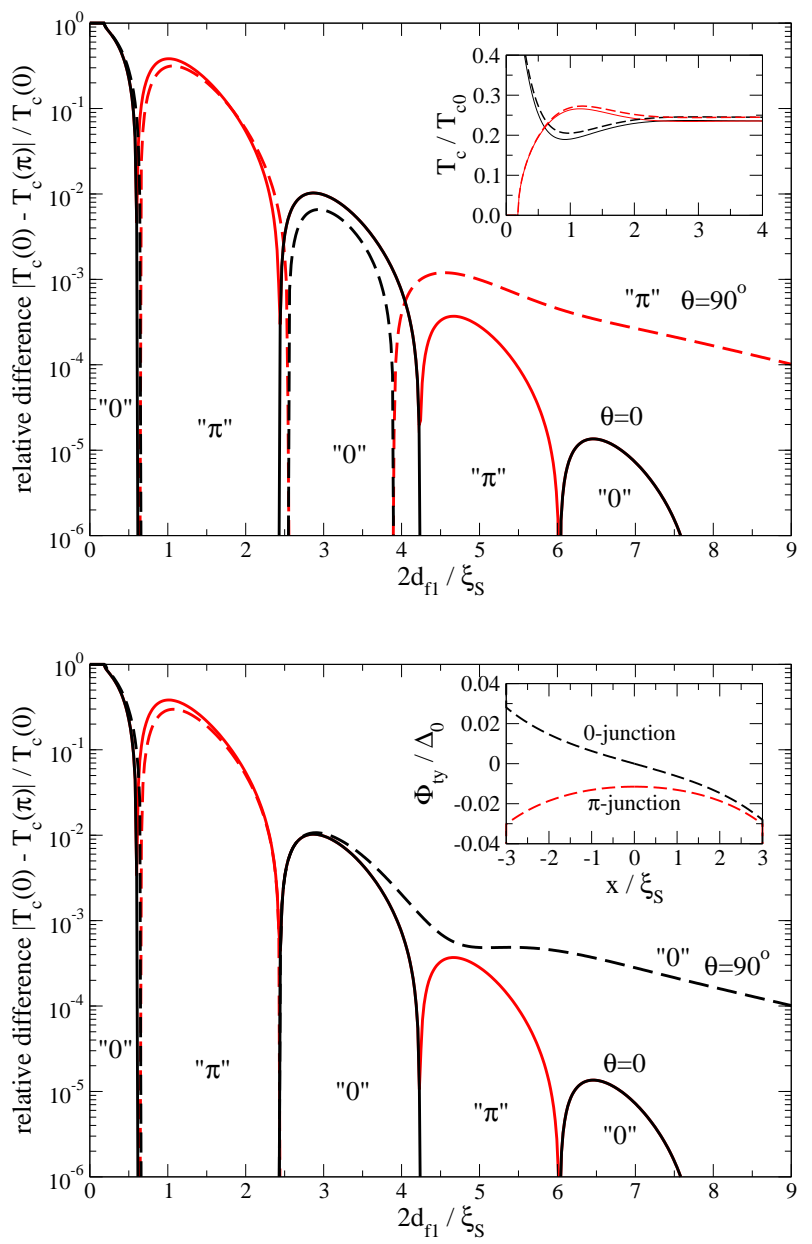

FIG. 14: For thick center films (large $d_{f 1}$ ) the communication between the two superconductors is taken over by long-range non-oscillatory equal spin triplet correlations and the junction is stabilized at phase difference 0 or $\pi$ depending on the exchange field orientation: the upper panel $(\pi$-junction for $\left.\theta=90^{\circ}\right)$ is obtained for exchange field rotation type $1(+\theta$ in both the left and right outer ferromagnets $\mathrm{F}_{2}$, as illustrated in Fig. 8), while the lower panel (0-junction for $\left.\theta=90^{\circ}\right)$ is obtained for rotation type $2\left(-\theta\right.$ in the left $\mathrm{F}_{2}$ and $+\theta$ in the right $\mathrm{F}_{2}$ ). The difference in $T_{c}$ between the 0 and $\pi$ cases is quite small for large $d_{f 1}$. Upper inset: the differences in $T_{c}$ for various exchange field orientations (solid lines $\theta=0$, dashed lines $\theta=90^{\circ}$ ) are due to the interaction between the ferromagnetic layers $F_{1}$ and $F_{2}$. Lower inset: spatial dependence inside the central $6 \xi_{s}$ thick $\mathrm{F}_{1}$ layer of the long-range triplet $f_{t y}$ induced for $\theta=90^{\circ}$. The parameters are $d_{s}=2 \xi_{s}$, $d_{f 2}=0.5 \xi_{s}, d_{f 1}=3 \xi_{s}, \gamma_{1}=\gamma_{2}=0.3, \gamma_{b 1}=\gamma_{b 2}=0.8$ and $J_{1}=J_{2}=10 T_{c 0}$.

has been found in Ref. 37 from calculations of the critical current in S-F multilayered junctions). We consider two types of rotation: the exchange fields in the outer ferromagnets are rotated by $+\theta$ as in Fig. 8 (rotation type 1 , $+\theta /+\theta)$, or rotations by $-\theta$ and $+\theta$ in the left and right outer layers respectively (rotation type $2,-\theta /+\theta$ ). The only difference between the two phase differences 0 and $\pi$ is the parity property of one of the triplets: $f_{t y}$ is an even 
(odd) function of $x$ for the 0 -junction ( $\pi$-junction) for rotation type $1(+\theta /+\theta)$. The parity properties of $f_{t y}$ for 0 and $\pi$ phase differences are reversed for rotation type $2(-\theta /+\theta)$. When $f_{t y}$ has odd parity it is smaller compared to the even parity case, which leads to a smaller suppression of the singlet $f_{s}$, i.e. less pair breaking, and a higher $T_{c}$. We therefore have a $\pi$-junction at large $d_{f 1}$ for rotation type 1 (upper panel in Fig. (14), and a 0-junction for rotation type 2 (lower panel in Fig. 114). We show the spatial dependence of the long range (in the central $F_{1}$ layer) triplet Green's function

$$
\Phi_{t y}(x)=T \sum_{\varepsilon_{n}>0} f_{t y}\left(\varepsilon_{n}, x\right)
$$

in the lower inset of Fig. 14.

Experimentally, the transition from $0 \rightarrow \pi$ was studied until now by varying the thickness 17.29 .30 .31 .32 of the ferromagnet in S-F-S junctions, or by varying the temperature 26.27 .28 which is more practical since the transition is seen in the same device. Here we have studied another possibility to switch from the 0 to the $\pi$ state within the same device, namely by continuously changing the relative orientation of the ferromagnetic moments. Our results are qualitatively consistent with the results obtained within Josephson critical current calculations $\stackrel{34.37}{{ }^{3}}$ The feasibility of controlling the orientation of the moments has been proven experimentally through the investigation of F-S-F trilayers for different moment orientations $\frac{16,19,20,21}{1}$

\section{DISCUSSION OF THE NUMERICS}

Let us discuss some delicate problems that need to be addressed when $T_{c}$ is computed in inhomogeneous structures. In particular, we will compare the two methods of computing $T_{c}$ : Eq. (12) which we call the Fourier method and Eq. (9) which we call the grid method.

The most important problem to address in any calculation using Usadel's approximation is the fact that the Matsubara sum in Eq. (5) is intrinsically slowly convergent, as compared to calculations done with the more general Eilenberger approach. As we show in Appendix $\mathbb{E}$ the difference $f_{s}\left(\varepsilon_{n}\right)-\pi \Delta /\left|\varepsilon_{n}\right|$ appearing in the gap equation (5) is at high-energies proportional to $1 / \varepsilon_{n}^{2}$ for inhomogeneous systems. This can be contrasted with an Eilenberger approach, where the high-energy asymptotic is $1 /\left|\varepsilon_{n}\right|^{3}$. It is therefore always necessary to extend the Matsubara sum to high energies when the Usadel approximation is employed, see the dashed line in Fig. 15] In the example we need a technical cut-off of order $1000 T_{c 0}$ to compute $T_{c}$ with an accuracy of $1 \%$. However, since the high-energy form of $f_{s}\left(\varepsilon_{n}\right)$ is known (see Appendix $\mathbb{E}$ ) it is in principle possible to circumvent the problem by treating the high-energy tail separately and sum the Matsubara sum to infinity. We have done that within the Fourier series approach, see Appendix $\mathbf{E}$
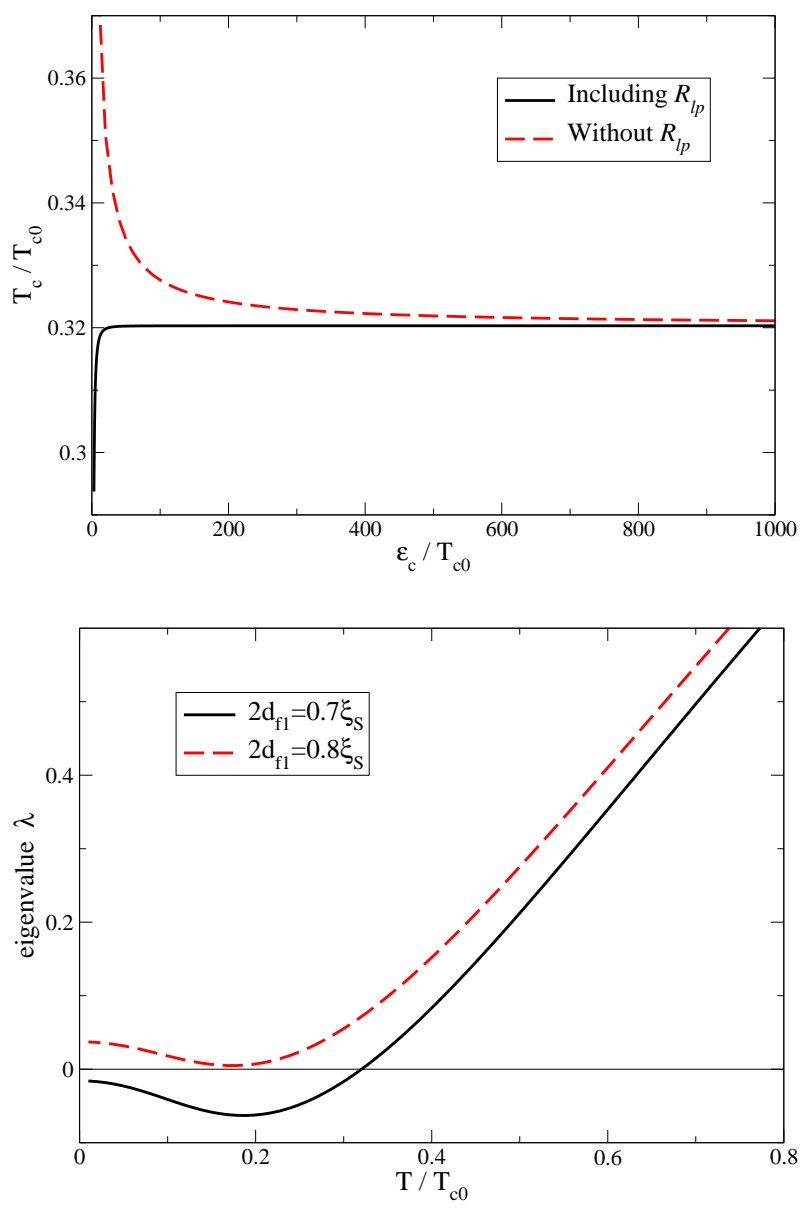

FIG. 15: Upper panel: critical temperature versus the technical cut-off $\varepsilon_{c}$. To achieve good accuracy for the critical temperature we need $\varepsilon_{c}$ of order $1000 T_{c 0}$ (dashed line). When the high-energy tail is summed to infinity, as described for the Fourier method in Eq. F10, the convergence is more acceptable (solid line). Lower panel: the eigenvalue of the gap equation (12) versus temperature for two different thicknesses. The zero-crossing determines $T_{c}$. When $T_{c}$ is suppressed, $\lambda(T)$ can become a flat function of $T$ which makes it important to compute $\lambda$ with high accuracy to avoid numerical errors in $T_{c}$. The parameters in the upper panel were chosen as in Fig. 113 0 -junction, at $2 d_{f 1}=0.7$ and $\theta=0$. In the lower panel the two thicknesses are indicated in the legend.

A more acceptable cut-off of order $100 T_{c}$ is then enough to achieve excellent accuracy, see the solid line in Fig. [15]

There are several other factors that, together with the slow convergence of the Matsubara sum, conspire to make it non-trivial to achieve acceptable accuracy, especially when $T_{c}$ is small compared to $T_{c 0}$. The critical temperature is computed by finding the temperature for which the eigenvalue $\lambda$ of the gap equation is zero [Fourier method, Eq. (12)] or one [grid method, Eq. (92)]. The function $\lambda(T)$ can become a very flat function of $T$ in the region where $T_{c 0}$ is small, see the lower panel of Fig. [15] Any error made in the calculation of $\lambda$ can therefore be magnified to a larger error in $T_{c}$ and it becomes increas- 
ingly critical to compute $\lambda$ with high accuracy as $T_{c}$ is suppressed.

The above two technical problems are particularly hard to circumvent within the grid method. First of all, the need to include high energies up to a technical cut-off $\varepsilon_{c}$ imposes a condition on the grid spacing $\delta x$. At high energies the function $G\left(\varepsilon_{n}, x, y\right)$ is typically peaked in the region $x \sim y$

$$
G\left(\varepsilon_{n} \gg T_{c 0}, x, y\right) \sim \frac{k_{s}\left(\varepsilon_{n}\right)}{\varepsilon_{n}} e^{-k_{s}\left(\varepsilon_{n}\right)|x-y|},
$$

where $k_{s}\left(\varepsilon_{n}\right)=\sqrt{2 \varepsilon_{n} / D}$. It is therefore necessary to choose

$$
\frac{\delta x}{\xi_{S}} \ll \frac{1}{\xi_{S} k_{s}\left(\varepsilon_{c}\right)}=\sqrt{\frac{\pi T_{c 0}}{\varepsilon_{c}}},
$$

to resolve this dependence. Since we need a cut-off around $1000 T_{c 0}$, because of the slow convergence within the Usadel approach, we need a grid spacing of order $0.01 \xi_{S}$ or finer. The matrix in Eq. (9) must therefore typically be of the order of a few hundred elements square, which severely slows down the numerics.

One reason for the importance to resolve the peaked form of $G\left(\varepsilon_{n}, x, y\right)$ is due to the interchange in order of the Matsubara sum and the integration over $y$ in Eq. (9). We write Eq. (9) as

$$
\int_{0}^{d_{s}} K(x, y) \Delta(y) d y=\Delta(x),
$$

and compute each element of the matrix $K(x, y)$ by summing over $\varepsilon_{n}$. The asymptotic form of the diagonal is however $G\left(\varepsilon_{n}, x, x\right) \propto 1 / \sqrt{\varepsilon_{n}}$ and the Matsubara sum is not convergent. This is in principle irrelevant for the calculation of $T_{c}$ because $T_{c}$ only depends on the eigenvalue of the matrix, which is a quantity given by the Matsubara sum integrated over $y$. Note that when Eq. (18) is integrated over $y$, a factor $1 / k_{s}$ appears in the primitive function of the exponential and the asymptotic form is $1 /\left|\varepsilon_{n}\right|$, which is (by construction) cancelled by the sum over $1 /\left|\varepsilon_{n}\right|$ in the denominator of $K(x, y)$, see Eq. (9). Numerically, however, the integral over the discretized coordinate $y$ can only be computed with some accuracy given by the grid spacing $\delta x$. The error made in computing the integral is transferred into an error in the eigenvalue $\lambda$ which, as described above, can result in an error in $T_{c}$ magnified by the flatness of the $\lambda(T)$-dependence.

To circumvent the problems described above, one must predict the high-energy tail to avoid cut-offs larger than $\sim 100 T_{c 0}$. Within the grid-method that means computing the derivative of $\Delta(x)$, i.e. to introduce an approximate formula for the derivative on a discretized grid. But that also introduces numerical errors and the grid must still be dense, which means that the matrix $K(x, y)$ remains large and the calculation with the grid method is always very slow and susceptible to numerical errors.

All the problems related to the discretization of the spatial coordinate are avoided within the Fourier series

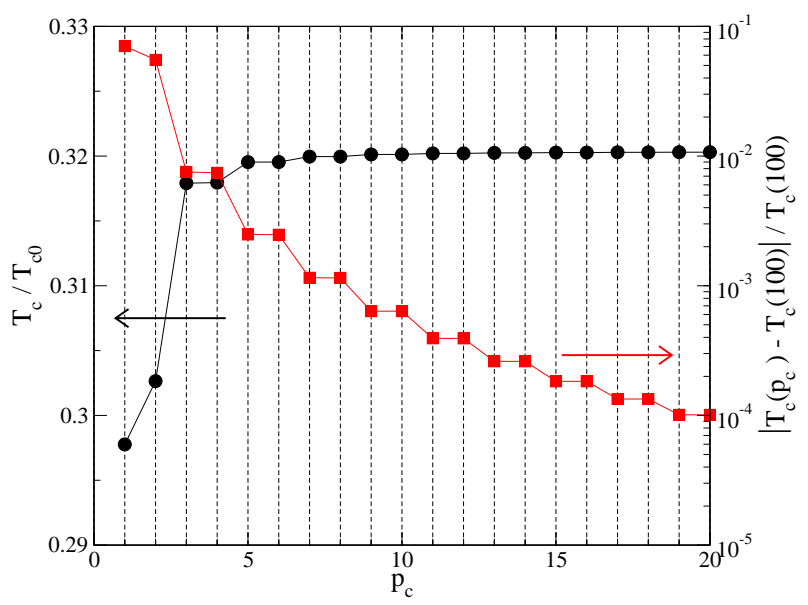

FIG. 16: Critical temperature versus the number $p_{c}$ of included Fourier coefficients in Eq. (10). The variation in absolute numbers are shown by circles (vertical scale to the left), while the variation in relation to the corresponding value for $T_{c}$ at a high cut-off $p_{c}=100$ is shown by squares (vertical scale to the right). The even-odd variation is due to the choice of parameters: the junction is almost symmetric and the evennumber Fourier components corresponding to symmetric cosfunctions contribute more to $T_{c}$. The model parameters were chosen as in Fig. 15 upper panel.

approach, since $G\left(\varepsilon_{n}, x, y\right)$ is analytically integrated over $x$ and $y$ in the course of the derivation of the matrix $m_{l p}$ in Eq. (12), see Appendix D Moreover, the high-energy tail is easily predicted analytically, see Appendix $\mathbf{E}$ It is typically sufficient to include only the 20 first Fourier components in the calculation of $T_{c}$, see Fig. 16] The matrix $m_{l p}$ is therefore small, the high-energy cut-off of the Matsubara sum can be chosen reasonably small, and very high accuracy is achieved while the speed of the calculation remains very high.

\section{SUMMARY}

In conclusion, we have studied the change of the superconducting critical temperature, $T_{c}$, in asymmetric trilayers $\mathrm{F}_{1}-\mathrm{S}-\mathrm{F}_{2}$ and symmetric pentalayers $\mathrm{F}_{2}-\mathrm{S}-\mathrm{F}_{1}-\mathrm{S}-\mathrm{F}_{2}$ with any relative orientation angle between the magnetizations of $\mathrm{F}_{1}$ and $\mathrm{F}_{2}$. For both cases we have presented phase diagrams, showing $T_{c}$ as function of the misorientation angle, $\theta$, and as a function of the ferromagnet layer thicknesses. We have investigated the interplay of longrange triplet components and Josephson coupling in the pentalayer geometry. We have demonstrated the possibility to switch between the 0 and $\pi$ states by controlling the relative orientation of the $\mathrm{F}$ moments in a pentalayer structure. This behavior may be appealing for the experimental study of the $0 \rightarrow \pi$ transition. We have presented details for a general method for the computation of $T_{c}$ and the dependence of the order parameter on the spatial coordinates in diffusive hybrid structures. 
With this technique, the accuracy as well as the speed of the numerics are immensely improved compared with previously used techniques.

\section{Acknowledgments}

We acknowledge support from the Deutsche Forschungsgemeinschaft within the Center for Functional Nanostructures (T.C., M.E.), and the Alexander von Humboldt Foundation (T.L.).

\section{APPENDIX A: DERIVATION OF $\hat{W}$ FOR THE TRILAYER}

In this Appendix we provide the details of the calculations leading to the effective boundary condition (6) obeyed by the singlet component in the superconducting region in asymmetric $\mathrm{F}_{1}-\mathrm{S}-\mathrm{F}_{2}$ trilayers with an arbitrary mutual orientation between the magnetizations in $F_{1}$ and $F_{2}$. Except for this latter component, it is possible to derive analytically the spatial dependences of all the components of the anomalous Green's function $f$ close to $T_{c}$ (next Section). In Section A 2 we determine from the consideration of the boundary conditions (3)-(4) the matrix $\hat{W}$ that enters the expression for the effective boundary condition (6).

\section{Spatial Dependences}

In the superconducting layer, the triplet vector $\mathbf{f}_{t}$ obeys a homogeneous differential equation [Eq. (2) with $\mathbf{J}=\mathbf{0}$ ] which is straightforwardly solved:

$$
\mathbf{f}_{t}=\mathbf{c} \cosh \left(k_{s} x\right)+\mathbf{d} \sinh \left(k_{s} x\right)
$$

with $\mathbf{c}$ and $\mathbf{d}$ constants.

For a fixed exchange field in each $\mathrm{F}$ layer, the system of coupled Eqs. (11)-(2) can be easily solved in the ferromagnetic regions. After application of the boundary conditions at the outer surfaces, the solutions can be written in the form 42

$$
\left(\begin{array}{c}
f_{s} \\
\mathbf{f}_{t}
\end{array}\right)=\sum_{\varepsilon= \pm} a_{\varepsilon} \cosh \left[k_{\varepsilon 1}\left(x+d_{f 1}\right]\left(\begin{array}{c}
1 \\
\varepsilon \hat{\mathbf{z}}
\end{array}\right)+a_{0} \cosh \left[k_{01}\left(x+d_{f 1}\right)\right]\left(\begin{array}{c}
0 \\
\hat{\mathbf{y}}
\end{array}\right)\right.
$$

for the $\mathrm{F}_{1}$ layer, and

$$
\left(\begin{array}{c}
f_{s} \\
\mathbf{f}_{t}
\end{array}\right)=\sum_{\varepsilon= \pm} b_{\varepsilon} \cosh \left[k_{\varepsilon 2}\left(x-d_{s}-d_{f 2}\right)\right]\left(\begin{array}{c}
1 \\
\varepsilon(\cos \theta \hat{\mathbf{z}}+\sin \theta \hat{\mathbf{y}})
\end{array}\right)+b_{0} \cosh \left[k_{02}\left(x-d_{s}-d_{f 2}\right)\right]\left(\begin{array}{c}
0 \\
\sin \theta \hat{\mathbf{z}}-\cos \theta \hat{\mathbf{y}}
\end{array}\right) A_{3}
$$

for the $\mathrm{F}_{2}$ layer. Here we have defined

$$
\begin{aligned}
& k_{ \pm q}=\sqrt{\left(2 \varepsilon_{n} \pm 2 i J_{q}\right) / D_{f q}}, \\
& k_{0 q}=\sqrt{2 \varepsilon_{n} / D_{f q}},
\end{aligned}
$$

with the index $q=1$ or 2 referring to the $\mathrm{F}_{1}$ or $\mathrm{F}_{2}$ layer.

\section{Determination of $\hat{W}$}

The constants $a_{j}$ and $b_{j}(j= \pm, 0)$, c and $\mathbf{d}$ are determined with the help of the boundary conditions (3)-(4) considered for the two $\mathrm{S} / \mathrm{F}$ interfaces. Writing these conditions for the triplet components only, we have

$$
\begin{array}{r}
\xi_{s} f_{l}^{\prime}\left(x_{S q}\right)=\gamma_{q} \xi_{f q} f_{l}^{\prime}\left(x_{F q}\right), \\
f_{l}\left(x_{S q}\right)=f_{l}\left(x_{F q}\right)+\eta_{q} \gamma_{b q} \xi_{f q} f_{l}^{\prime}\left(x_{F q}\right),
\end{array}
$$

with $l=t_{y}, t_{z}$. Note that $\eta_{1}=+1$ and $\eta_{2}=-1$. Similarly, we get for the singlet amplitude

$$
\begin{array}{r}
\xi_{s} f_{s}^{\prime}\left(x_{S q}\right)=\gamma_{q} \xi_{f q} f_{s}^{\prime}\left(x_{F q}\right), \\
f_{s}\left(x_{S q}\right)=f_{s}\left(x_{F q}\right)+\eta_{q} \gamma_{b q} \xi_{f q} f_{s}^{\prime}\left(x_{F q}\right) .
\end{array}
$$


Here, $x_{F 1}$ and $x_{S 1}$ are the coordinates on the two sides of the $\mathrm{F}_{1} / \mathrm{S}$ interface at $x_{1}=0$, while $x_{F 2}$ and $x_{S 2}$ refers to the $\mathrm{S} / \mathrm{F}_{2}$ interface at $x_{2}=d_{s}$. From Eqs. (A9) and (A7) for the first interface $\left(\mathrm{F}_{1} / \mathrm{S}\right)$, we obtain the system

$$
\begin{aligned}
f_{s}\left(x_{1}\right) & =\sum_{\varepsilon= \pm} a_{\varepsilon} \mathcal{A}_{\varepsilon}, \\
c_{y} & =a_{0} \mathcal{A}_{0}, \\
c_{z} & =\sum_{\varepsilon= \pm} \varepsilon a_{\varepsilon} \mathcal{A}_{\varepsilon},
\end{aligned}
$$

with the quantity

$$
\mathcal{A}_{j}=\cosh \left(k_{j 1} d_{f 1}\right)+\gamma_{b 1} k_{j 1} \xi_{f 1} \sinh \left(k_{j 1} d_{f 1}\right)
$$

where $j= \pm, 0$. The matching of the different components with the conditions a99 and (A7) yield at the second interface $\left(\mathrm{S} / \mathrm{F}_{2}\right)$

$$
\begin{aligned}
f_{s}\left(x_{2}\right) & =\sum_{\varepsilon= \pm} b_{\varepsilon} \mathcal{B}_{\varepsilon}, \\
c_{y} \cosh \left(k_{s} d_{s}\right)+d_{y} \sinh \left(k_{s} d_{s}\right) & =\sum_{\varepsilon= \pm} \varepsilon b_{\varepsilon} \mathcal{B}_{\varepsilon} \sin \theta-b_{0} \mathcal{B}_{0} \cos \theta, \\
c_{z} \cosh \left(k_{s} d_{s}\right)+d_{z} \sinh \left(k_{s} d_{s}\right) & =\sum_{\varepsilon= \pm} \varepsilon b_{\varepsilon} \mathcal{B}_{\varepsilon} \cos \theta+b_{0} \mathcal{B}_{0} \sin \theta,
\end{aligned}
$$

with

$$
\mathcal{B}_{j}=\cosh \left(k_{j 2} d_{f 2}\right)+\gamma_{b 2} k_{j 2} \xi_{f 2} \sinh \left(k_{j 2} d_{f 2}\right)
$$

defined in a similar way as the quantity $\mathcal{A}_{j}$. Then, the boundary conditions y6 yield the system

$$
\begin{aligned}
d_{y} & =a_{0} \mathcal{C}_{0}, \\
d_{z} & =\sum_{\varepsilon= \pm} \varepsilon a_{\varepsilon} \mathcal{C}_{\varepsilon},
\end{aligned}
$$

for the $\mathrm{F}_{1} / \mathrm{S}$ interface, and

$$
\begin{aligned}
& c_{y} \sinh \left(k_{s} d_{s}\right)+d_{y} \cosh \left(k_{s} d_{s}\right)=b_{0} \mathcal{D}_{0} \cos \theta-\sum_{\varepsilon= \pm} \varepsilon b_{\varepsilon} \mathcal{D}_{\varepsilon} \sin \theta, \\
& c_{z} \sinh \left(k_{s} d_{s}\right)+d_{z} \cosh \left(k_{s} d_{s}\right)=-b_{0} \mathcal{D}_{0} \sin \theta-\sum_{\varepsilon= \pm} \varepsilon b_{\varepsilon} \mathcal{D}_{\varepsilon} \cos \theta,
\end{aligned}
$$

for the $\mathrm{S} / \mathrm{F}_{2}$ interface, with

$$
\begin{aligned}
& \mathcal{C}_{j}=\gamma_{1} k_{j 1} \xi_{f 1} \sinh \left(k_{j 1} d_{f 1}\right) / k_{s} \xi_{s} \\
& \mathcal{D}_{j}=\gamma_{2} k_{j 2} \xi_{f 2} \sinh \left(k_{j 2} d_{f 2}\right) / k_{s} \xi_{s} .
\end{aligned}
$$

The next step consists of eliminating the coefficients $c_{y}, c_{z}, d_{y}, d_{z}, a_{0}, b_{0}$ from the former equations. We obtain the system

$$
\begin{aligned}
\sum_{\varepsilon} \varepsilon a_{\varepsilon} \mathcal{E}_{\varepsilon} & =\sum_{\varepsilon} \varepsilon b_{\varepsilon} \mathcal{G}_{\varepsilon}, \\
\sum_{\varepsilon} \varepsilon b_{\varepsilon} \mathcal{F}_{\varepsilon} & =\sum_{\varepsilon} \varepsilon a_{\varepsilon} \mathcal{H}_{\varepsilon},
\end{aligned}
$$

where

$$
\begin{aligned}
\mathcal{E}_{\varepsilon} & =K_{0}\left(\mathcal{A}_{\varepsilon}-\mathcal{C}_{\varepsilon}\right)\left[\cosh \left(k_{s} d_{s}\right)-\sinh \left(k_{s} d_{s}\right)\right] \cos \theta, \\
\mathcal{F}_{\varepsilon} & =K_{\varepsilon}\left(\mathcal{B}_{0}-\mathcal{D}_{0}\right) \sin ^{2} \theta+K_{0}\left(\mathcal{B}_{\varepsilon}-\mathcal{D}_{\varepsilon}\right) \cos ^{2} \theta, \\
\mathcal{G}_{\varepsilon} & =K_{\varepsilon}\left(\mathcal{B}_{0}+\mathcal{D}_{0}\right) \sin ^{2} \theta+K_{0}\left(\mathcal{B}_{\varepsilon}+\mathcal{D}_{\varepsilon}\right) \cos ^{2} \theta, \\
\mathcal{H}_{\varepsilon} & =K_{0}\left(\mathcal{A}_{\varepsilon}+\mathcal{C}_{\varepsilon}\right)\left[\cosh \left(k_{s} d_{s}\right)+\sinh \left(k_{s} d_{s}\right)\right] \cos \theta,
\end{aligned}
$$


with

$$
K_{j}=\left[\mathcal{B}_{j} \mathcal{C}_{0}+\mathcal{D}_{j} \mathcal{A}_{0}\right] \cosh \left(k_{s} d_{s}\right)+\left[\mathcal{B}_{j} \mathcal{A}_{0}+\mathcal{D}_{j} \mathcal{C}_{0}\right] \sinh \left(k_{s} d_{s}\right)
$$

Compiling Eqs. (A10) and A14 with Eqs. A24 - A25e get the expressions for the amplitudes $a_{\varepsilon}$ and $b_{\varepsilon}$

$$
\begin{aligned}
a_{\varepsilon} & =\frac{\left(\mathcal{B}_{+} \mathcal{I}_{-,-\varepsilon}+\mathcal{B}_{-} \mathcal{I}_{+,-\varepsilon}\right) f_{s}\left(x_{1}\right)+\varepsilon \mathcal{A}_{-\varepsilon}\left(\mathcal{F}_{-} \mathcal{G}_{+}-\mathcal{F}_{+} \mathcal{G}_{-}\right) f_{s}\left(x_{2}\right)}{\mathcal{J}} \\
b_{\varepsilon} & =\frac{\left(\mathcal{A}_{+} \mathcal{I}_{-\varepsilon,-}+\mathcal{A}_{-} \mathcal{I}_{-\varepsilon,+}\right) f_{s}\left(x_{2}\right)+\varepsilon \mathcal{B}_{-\varepsilon}\left(\mathcal{E}_{-} \mathcal{H}_{+}-\mathcal{E}_{+} \mathcal{H}_{-}\right) f_{s}\left(x_{1}\right)}{\mathcal{J}},
\end{aligned}
$$

with

$$
\begin{aligned}
\mathcal{I}_{\varepsilon, \varepsilon^{\prime}} & =\mathcal{F}_{\varepsilon} \mathcal{E}_{\varepsilon^{\prime}}-\mathcal{G}_{\varepsilon} \mathcal{H}_{\varepsilon^{\prime}} \\
\mathcal{J} & =\mathcal{A}_{+} \mathcal{B}_{+} \mathcal{I}_{-,-}+\mathcal{A}_{-} \mathcal{B}_{-} \mathcal{I}_{+,+}+\mathcal{A}_{+} \mathcal{B}_{-} \mathcal{I}_{+,-}+\mathcal{A}_{-} \mathcal{B}_{+} \mathcal{I}_{-,+} .
\end{aligned}
$$

Finally, Eqs. A8 yield the system

$$
\begin{aligned}
& \xi_{s} f_{s}^{\prime}\left(x_{1}\right)=k_{s} \xi_{s} \sum_{\varepsilon} a_{\varepsilon} \mathcal{C}_{\varepsilon}, \\
& \xi_{s} f_{s}^{\prime}\left(x_{2}\right)=-k_{s} \xi_{s} \sum_{\varepsilon} b_{\varepsilon} \mathcal{D}_{\varepsilon}
\end{aligned}
$$

which can be rewritten in the form

$$
\left(\begin{array}{c}
f_{s}^{\prime}\left(x_{1}\right) \\
f_{s}^{\prime}\left(x_{2}\right)
\end{array}\right)=k_{s}\left(\begin{array}{ll}
W_{11} & W_{12} \\
W_{21} & W_{22}
\end{array}\right)\left(\begin{array}{l}
f_{s}\left(x_{1}\right) \\
f_{s}\left(x_{2}\right)
\end{array}\right)
$$

with

$$
\begin{aligned}
W_{11} & =\frac{\mathcal{C}_{+}\left(\mathcal{B}_{+} \mathcal{I}_{-,-}+\mathcal{B}_{-} \mathcal{I}_{+,-}\right)+\mathcal{C}_{-}\left(\mathcal{B}_{+} \mathcal{I}_{-,+}+\mathcal{B}_{-} \mathcal{I}_{+,+}\right)}{\mathcal{J}}, \\
W_{22} & =-\frac{\mathcal{D}_{+}\left(\mathcal{A}_{+} \mathcal{I}_{-,-}+\mathcal{A}_{-} \mathcal{I}_{-,+}\right)+\mathcal{D}_{-}\left(\mathcal{A}_{+} \mathcal{I}_{+,-}+\mathcal{A}_{-} \mathcal{I}_{+,+}\right)}{\mathcal{J}}, \\
W_{12} & =\frac{\left(\mathcal{F}_{-} \mathcal{G}_{+}-\mathcal{F}_{+} \mathcal{G}_{-}\right)\left(\mathcal{A}_{-} \mathcal{C}_{+}-\mathcal{A}_{+} \mathcal{C}_{-}\right)}{\mathcal{J}}, \\
W_{21} & =-\frac{\left(\mathcal{E}_{-} \mathcal{H}_{+}-\mathcal{E}_{+} \mathcal{H}_{-}\right)\left(\mathcal{B}_{-} \mathcal{D}_{+}-\mathcal{B}_{+} \mathcal{D}_{-}\right)}{\mathcal{J}}
\end{aligned}
$$

Using the expressions A26 - one can notice that in fact $W_{12}=-W_{21}$ with

$$
W_{12}=\frac{2 K_{0}^{2} \cos ^{2} \theta\left(\mathcal{B}_{-} \mathcal{D}_{+}-\mathcal{B}_{+} \mathcal{D}_{-}\right)\left(\mathcal{A}_{-} \mathcal{C}_{+}-\mathcal{A}_{+} \mathcal{C}_{-}\right)}{\mathcal{J}}
$$

For an asymmetric trilayer $\mathrm{F}_{1}-\mathrm{S}-\mathrm{F}_{2}$, the diagonal coefficients $W_{11}$ and $W_{22}$ of the matrix $\hat{W}$ differ in general. In the special case of a symmetric trilayer $\mathrm{F}_{1}-\mathrm{S}_{-} \mathrm{F}_{1}$, we have $W_{11}=W_{22}$.

\section{APPENDIX B: DERIVATION OF $\hat{W}$ FOR THE PENTALAYER}

Due to the symmetry of the geometry, we need to determine the components of the anomalous Green function $f$ only in half of the pentalayer, e.g. in the domain $x>0$. The problem is mapped back onto the asymmetrical $\mathrm{F}_{1}$ $\mathrm{S}-\mathrm{F}_{2}$ trilayer problem previously considered in Appendix A. Because we have chosen a different origin for the system of coordinates, the $\mathrm{F}_{1} / \mathrm{S}$ and $\mathrm{S} / \mathrm{F}_{2}$ interfaces are now located at the positions $x_{1}=d_{f 1}$ and $x_{2}=d_{s}+d_{f 1}$. Due to the shift in coordinates, we have used the expressions (A1) in the $\mathrm{S}$ layer and (A3) in the $\mathrm{F}_{2}$ layer with $x$ replaced by $x-d_{f 1}$.

For the rotation type 1 , the spatial dependences of the singlet and triplet components of $f$ in the left $\mathrm{F}_{1}$ layer are in the 0-junction case the same as in Eq. (A2) after the shift of coordinate. In the $\pi$-junction case, the boundary conditions at the (fictitious) outer surface $x=0$ have changed, and the spatial dependences in $\mathrm{F}_{1}$ are now given 
by:

$$
\left(\begin{array}{c}
f_{s} \\
\mathbf{f}_{t}
\end{array}\right)=\sum_{\varepsilon= \pm} a_{\varepsilon} \sinh \left[k_{\varepsilon} x\right]\left(\begin{array}{c}
1 \\
\varepsilon \hat{\mathbf{z}}
\end{array}\right)+a_{0} \sinh \left[k_{01} x\right]\left(\begin{array}{c}
0 \\
\hat{\mathbf{x}}
\end{array}\right) .
$$

The new boundary conditions (13) and (14) at $x=0$ do not affect the definition of the former quantities $\mathcal{B}_{j}$ and $\mathcal{D}_{j}$. On the other hand, changes occur in the definition of the quantities $\mathcal{A}_{j}$ and $\mathcal{C}_{j}$ (where $j=\varepsilon$ or 0 ). In the 0 -junction case, the coefficients $\mathcal{A}_{j}$ and $\mathcal{C}_{j}$ remain unchanged, while in the $\pi$-junction case they are defined as

$$
\begin{aligned}
\mathcal{A}_{j} & =\sinh \left(k_{j 1} d_{f 1}\right)+\gamma_{b 1} k_{j 1} \xi_{f 1} \cosh \left(k_{j 1} d_{f 1}\right), \\
\mathcal{C}_{j} & =\gamma_{1} k_{j 1} \xi_{f 1} \cosh \left(k_{j 1} d_{f 1}\right) / k_{s} \xi_{s} .
\end{aligned}
$$

For the rotation type 2 , the components of $f$ in $\mathrm{F}_{1}$ have a different spatial dependence as a result of the conditions (15) or (16). They are expressed as

$$
\left(\begin{array}{c}
f_{s} \\
\mathbf{f}_{t}
\end{array}\right)=\sum_{\varepsilon= \pm} a_{\varepsilon} \cosh \left[k_{\varepsilon 1} x\right]\left(\begin{array}{c}
1 \\
\varepsilon \hat{\mathbf{z}}
\end{array}\right)+a_{0} \sinh \left[k_{01} x\right]\left(\begin{array}{c}
0 \\
\hat{\mathbf{x}}
\end{array}\right)
$$

in the 0 -junction case, and

$$
\left(\begin{array}{c}
f_{s} \\
\mathbf{f}_{t}
\end{array}\right)=\sum_{\varepsilon= \pm} a_{\varepsilon} \sinh \left[k_{\varepsilon 1} x\right]\left(\begin{array}{c}
1 \\
\varepsilon \hat{\mathbf{z}}
\end{array}\right)+a_{0} \cosh \left[k_{01} x\right]\left(\begin{array}{c}
0 \\
\hat{\mathbf{x}}
\end{array}\right)
$$

in the $\pi$-junction case. As for rotation type 1 , changes occur in the definition of the quantities $\mathcal{A}_{j}$ and $\mathcal{C}_{j}$ (where $j=\varepsilon$ or 0 ) for the rotation type 2 . In the 0 -junction case, the coefficients $\mathcal{A}_{\varepsilon}$ and $\mathcal{C}_{\varepsilon}$ have the same expression as in Appendix $\mathrm{A}$ while $\mathcal{A}_{0}$ and $\mathcal{C}_{0}$ are now given by

$$
\begin{aligned}
\mathcal{A}_{0} & =\sinh \left(k_{01} d_{f 1}\right)+\gamma_{b 1} k_{j 1} \xi_{f 1} \cosh \left(k_{01} d_{f 1}\right), \\
\mathcal{C}_{0} & =\gamma_{1} k_{01} \xi_{f 1} \cosh \left(k_{01} d_{f 1}\right) / k_{s} \xi_{s} .
\end{aligned}
$$

In the $\pi$-junction case, the quantities $\mathcal{A}_{0}$ and $\mathcal{C}_{0}$ are defined in the same way as in Appendix while $\mathcal{A}_{\varepsilon}$ and $\mathcal{C}_{\varepsilon}$ are written as

$$
\begin{aligned}
\mathcal{A}_{\varepsilon} & =\sinh \left(k_{\varepsilon 1} d_{f 1}\right)+\gamma_{b 1} k_{\varepsilon 1} \xi_{f 1} \cosh \left(k_{01} d_{f 1}\right), \\
\mathcal{C}_{\varepsilon} & =\gamma_{1} k_{\varepsilon 1} \xi_{f 1} \cosh \left(k_{\varepsilon 1} d_{f 1}\right) / k_{s} \xi_{s} .
\end{aligned}
$$

Except for these modifications in the definition of the quantities $\mathcal{A}$ and $\mathcal{C}$, the remaining calculations are exactly the same as in the asymmetric trilayer geometry and we can use the final expression derived in Appendix A for the matrix $\hat{W}$ in the symmetric pentalayer structure.

\section{APPENDIX C: DERIVATION OF $G\left(\varepsilon_{n}, x, y\right)$}

In analogy with Ref. [8, Eq. (8) is solved by making the following ansatz

$$
G(x, y)= \begin{cases}L_{c}(y) X_{1}(x)+L_{s}(y) X_{2}(x), & x<y, \\ R_{c}(y) Y_{1}(x)+R_{s}(y) Y_{2}(x), & y<x,\end{cases}
$$

where we introduced the notation

$$
\begin{aligned}
X_{1}(x) & =\cosh \left(k_{s} x\right), \\
X_{2}(x) & =\sinh \left(k_{s} x\right), \\
Y_{1}(x) & =\cosh \left(k_{s}\left[x-d_{s}\right]\right), \\
Y_{2}(x) & =\sinh \left(k_{s}\left[x-d_{s}\right]\right) .
\end{aligned}
$$

The coefficients $L_{c}, L_{s}, R_{c}$ and $R_{s}$ depend on the location $y$ of the source term in Eq. (8). The source is taken into account by the conditions

$$
\left.G(x, y)\right|_{x=y^{+}}=\left.G(x, y)\right|_{x=y^{-}},
$$

and

$$
\left.\partial_{x} G(x, y)\right|_{x=y^{+}}-\left.\partial_{x} G(x, y)\right|_{x=y^{-}}=-k_{s}^{2} / \varepsilon_{n},
$$

where $y^{+}$and $y^{-}$denote the limits $y \rightarrow x$ from above and below, respectively. Eqs. [C6)-(C7) give two relations between the coefficients in Eq. (C1). Two additional relations are provided by the boundary conditions at the edges of the superconductor, which read

$$
\left(\begin{array}{c}
\left.\partial_{x} G(x, y)\right|_{x=0} \\
\left.\partial_{x} G(x, y)\right|_{x=d_{s}}
\end{array}\right)=k_{s} \hat{W}\left(\begin{array}{c}
G(0, y) \\
G\left(d_{s}, y\right)
\end{array}\right) .
$$

These conditions are consistent with the boundary conditions (6) obeyed by the singlet amplitude $f_{s}(x)$. Compiling Eqs. (C1)-(C8), we obtain the coefficients

$$
\begin{aligned}
L_{c}(y) & =\frac{k_{s}}{\varepsilon_{n} \mathcal{L}}\left[Y_{1}(y)+W_{22} Y_{2}(y)-W_{12} X_{2}(y)\right], \\
R_{c}(y) & =\frac{k_{s}}{\varepsilon_{n} \mathcal{L}}\left[X_{1}(y)-W_{21} Y_{2}(y)+W_{11} X_{2}(y)\right], \\
L_{s}(y) & =\frac{k_{s}}{\varepsilon_{n} \mathcal{L}}\left[W_{11} Y_{1}(y)+W_{12} X_{1}(y)+\operatorname{det}(\hat{W}) Y_{2}(y)\right], \\
R_{s}(y) & =\frac{k_{s}}{\varepsilon_{n} \mathcal{L}}\left[W_{21} Y_{1}(y)+W_{22} X_{1}(y)+\operatorname{det}(\hat{W}) X_{2}(y)\right],
\end{aligned}
$$

where

$$
\begin{aligned}
\mathcal{L}= & W_{12}-W_{21}+\left(W_{11}-W_{22}\right) \cosh \left(k_{s} d_{s}\right) \\
& +[1-\operatorname{det}(\hat{W})] \sinh \left(k_{s} d_{s}\right) .
\end{aligned}
$$

We note that the dependence on the Matsubara frequency $\varepsilon_{n}$ enters through $k_{s}$ and the four elements $W_{11}$, $W_{22}, W_{12}$, and $W_{22}$ of the $2 \times 2$ matrix $\hat{W}$ in the boundary condition.

\section{APPENDIX D: DERIVATION OF $m_{l p}$ IN EQ. (12)}

We insert the expansion (10) into Eq. (17), use the expression for $G\left(\varepsilon_{n}, x, y\right)$ derived in Appendix [C] and perform the integration over the spatial coordinate $y$. We obtain the singlet amplitude $f_{s}\left(\varepsilon_{n}, x\right)$ in terms of the Fourier coefficients $\Delta_{p}$ 


$$
\begin{aligned}
f_{s}\left(\varepsilon_{n}, x\right)=\frac{\pi}{\varepsilon_{n} \mathcal{L}} \sum_{p=0}^{\infty} \Delta_{p} \beta_{p}\{ & \mathcal{L} \cos \left(\frac{\pi p x}{d_{s}}\right)+\left[W_{21}+(-1)^{p} W_{22}\right] X_{1}(x)-\left[W_{11}+(-1)^{p} W_{12}\right] Y_{1}(x) \\
& \left.+\operatorname{det}(\hat{W})\left[(-1)^{p} X_{2}(x)-Y_{2}(x)\right]\right\}
\end{aligned}
$$

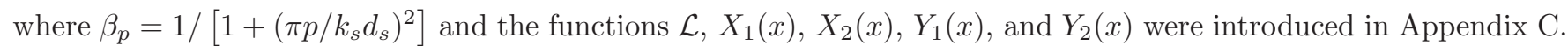
We insert this expression in the gap equation (9) and project in Fourier space, i.e. we multiply by $\cos \left(\pi l x / d_{s}\right)$ and integrate over $x$. As a result, we obtain a linear system for the Fourier components $\Delta_{p}$, with row $l \geq 0$ given by

$$
\sum_{p=0}^{+\infty} m_{l p} \Delta_{p}=0
$$

The off-diagonal elements $(l \neq p)$ have the form

$$
m_{l p}=4 \pi T \sum_{\varepsilon_{n}>0} \frac{1}{\varepsilon_{n}} b_{l p} \beta_{l} \beta_{p}
$$

while the diagonal elements $(l=p)$ are given by

$$
m_{l l}=\left(1+\delta_{l 0}\right) \ln \frac{T}{T_{c 0}}+4 \pi T \sum_{\varepsilon_{n}>0} \frac{1}{\varepsilon_{n}}\left[b_{l l} \beta_{l}^{2}+\frac{1}{2}\left(1-\beta_{l}\right)\right],
$$

where

$b_{l p}=\frac{\left[W_{11}-(-1)^{l+p} W_{22}+(-1)^{p} W_{12}-(-1)^{l} W_{21}\right] \sinh \left(k_{s} d_{s}\right)+\operatorname{det}(\hat{W})\left\{(-1)^{p}+(-1)^{l}-\left[1+(-1)^{l+p}\right] \cosh \left(k_{s} d_{s}\right)\right\}}{k_{s} d_{s} \mathcal{L}}$.

The relation $W_{12}=-W_{21}$ between the off-diagonal elements of $\hat{W}$ found in Appendix $\mathrm{A}$ implies that the matrix $\hat{m}$ is actually symmetric, i.e. $m_{l p}=m_{p l}$ (see expressions (D3) and (D5). This property guarantees the existence of real solutions of the eigenproblem (12).

\section{APPENDIX E: HIGH-ENERGY ASYMPTOTICS}

We present and compare the asymptotic high-energy behavior of the quasiclassical Green's function in the diffusive limit within the Usadel approximation to the more general case described by the Eilenberger equation. Since the present discussion is independent of the presence or absence of a weak exchange field $J \ll \epsilon_{f}$ in the system, we leave it out.

\section{Diffusive Limit}

The Usadel equation ${ }^{46}$ for arbitrary temperatures (not necessarily close to $T_{c}$ as in the rest of the paper) is

$$
\left[i \varepsilon_{n} \hat{\tau}_{3}-\hat{\Delta}, \hat{g}\right]+\frac{D}{\pi} \partial_{x}\left(\hat{g} \partial_{x} \hat{g}\right)=\hat{0},
$$

where $\hat{g}$ is a $4 \times 4$ matrix in combined particle-hole and spin spaces, $\hat{\tau}_{j}(j=1,2,3)$ are the Pauli matrices in particle-hole space, and $\hat{\Delta}$ is the gap function $\left(\hat{\Delta}=\left(i \sigma_{y}\right) \hat{\tau}_{1} \Delta\right.$ if $\Delta$ is real). Eq. (E1) is supplemented with a normalization condition

$$
\hat{g}^{2}=-\pi^{2} \hat{1} .
$$

Further details concerning the structure of the Green's function with the present notation can be found in Ref. 43 (see also Ref. 52).

At high energies the order parameter and the derivative term are small,

$$
\begin{aligned}
& \Delta \sim T_{c 0} \ll \varepsilon_{n}, \\
& D / \xi^{2} \sim T_{c 0} \ll \varepsilon_{n},
\end{aligned}
$$

and we expand the Green's function

$$
\hat{g}=\hat{g}^{(0)}+\hat{g}^{(1)}+\hat{g}^{(2)}+\ldots
$$

where the term $\hat{g}^{(k)}$ is of order $\left(T_{c 0} / \varepsilon_{n}\right)^{k}$. To lowest order we have

$$
\begin{aligned}
& {\left[i \varepsilon_{n} \hat{\tau}_{3}, \hat{g}^{(0)}\right]=0,} \\
& \left(\hat{g}^{(0)}\right)^{2}=-\pi^{2} \hat{1},
\end{aligned}
$$


with the solution

$$
\hat{g}^{(0)}=(-i \pi) \operatorname{sgn}\left(\varepsilon_{n}\right) \hat{\tau}_{3} .
$$

In first order we obtain

$$
\begin{aligned}
& {\left[i \varepsilon_{n} \hat{\tau}_{3}, \hat{g}^{(1)}\right]=\left[\hat{\Delta}, \hat{g}^{(0)}\right],} \\
& \hat{g}^{(0)} \hat{g}^{(1)}+\hat{g}^{(1)} \hat{g}^{(0)}=0 .
\end{aligned}
$$

Since $\hat{g}^{(0)}$ is proportional to $\hat{\tau}_{3}$, the second line can be used to move $\hat{g}^{(1)}$ to one side of the commutator on the left-hand-side of the first line. We obtain

$$
2 i \varepsilon_{n} \hat{\tau}_{3} \hat{g}^{(1)}=\left[\hat{\Delta}, \hat{g}^{(0)}\right] .
$$

The solution is purely off-diagonal in particle-hole space

$$
\hat{g}^{(1)}=\frac{(-i \pi)}{2 i\left|\varepsilon_{n}\right|}\left(\hat{\tau}_{3} \hat{\Delta} \hat{\tau}_{3}-\hat{\Delta}\right)=\frac{\pi}{\left|\varepsilon_{n}\right|} \hat{\Delta} .
$$

In second order we have

$$
\begin{aligned}
& {\left[i \varepsilon_{n} \hat{\tau}_{3}, \hat{g}^{(2)}\right]=-\frac{D}{\pi} \hat{g}^{(0)} \partial_{x}^{2} \hat{g}^{(1)},} \\
& \hat{g}^{(0)} \hat{g}^{(2)}+\hat{g}^{(2)} \hat{g}^{(0)}+\left(\hat{g}^{(1)}\right)^{2}=0 .
\end{aligned}
$$

After a short calculation, similar to the calculation in first order, we obtain

$$
\hat{g}^{(2)}=\frac{(-i \pi)}{2 \varepsilon_{n}\left|\varepsilon_{n}\right|} \hat{\tau}_{3} \hat{\Delta}^{2}+\frac{\pi D}{2 \varepsilon_{n}^{2}} \partial_{x}^{2} \hat{\Delta} .
$$

Note, in particular, that there is an off-diagonal term proportional to $1 / \varepsilon_{n}^{2}$ for inhomogeneous systems.

The off-diagonal part of the Green's function has according to the above the asymptotic form

$$
f\left(\varepsilon_{n}, x\right)=\frac{\pi \Delta(x)}{\left|\varepsilon_{n}\right|}+\frac{\pi D \partial_{x}^{2} \Delta(x)}{2 \varepsilon_{n}^{2}}+\mathcal{O}\left[\left(\frac{T_{c 0}}{\left|\varepsilon_{n}\right|}\right)^{3}\right],
$$

which we now use to discuss the gap equation. The gap equation

$$
\Delta(x)=\lambda T \sum_{\left|\varepsilon_{n}\right|<\omega_{p}} f\left(\varepsilon_{n}, x\right),
$$

contains a log-divergency and it is necessary to introduce a cut-off $\omega_{p}$. But by the well-known procedure (see e.g. Ref. [53), the interaction strength $\lambda$ and the Matsubara sum cut-off $\omega_{p}$ can both be eliminated by adding and subtracting the leading high-energy term in Eq. (E16). The gap equation then has the form in Eq. (5). The Matsubara sum converges, with a high-energy asymptotic tail $\propto 1 / \varepsilon_{n}^{2}$ according to Eq. (E16), and can be extended to infinity. In practice, a technical cut-off $\varepsilon_{c}$ is introduced that should, however, be high enough that the results of the calculation are cut-off independent.

\section{Arbitrary Mean Free Path}

We compare the above results obtained within the Usadel approximation with the corresponding high-energy behavior obtained within the Eilenberger approach. The Eilenberger equation ${ }^{54.55}$ reads

$$
\left[i \varepsilon_{n} \hat{\tau}_{3}-\hat{\Delta}-\hat{\sigma}_{i m p}, \hat{g}\right]+i \vec{v}_{F} \cdot \nabla \hat{g}=\hat{0},
$$

with impurity self energy $\hat{\sigma}_{i m p}$, and where $\vec{v}_{F}$ is the Fermi velocity. The normalization condition $\hat{g}^{2}=-\pi^{2} \hat{1}$ holds. We include non-magnetic impurity scattering within the self-consistent $t$-matrix approximation, for which the impurity self energy is

$$
\hat{\sigma}_{i m p}(s)=c \hat{t}(s, s),
$$

where $c$ is the impurity concentration, and $s$ is a parameter that specifies the position of the momentum on the Fermi surface. The $t$-matrix is given as the solution of the equation

$$
\begin{aligned}
\hat{t}\left(s, s^{\prime}\right)= & \hat{u}\left(s, s^{\prime}\right)+ \\
& \left\langle\hat{u}\left(s, s^{\prime \prime}\right) \mathcal{N}_{F}\left(s^{\prime \prime}\right) \hat{g}\left(s^{\prime \prime}\right) \hat{t}\left(s^{\prime \prime}, s^{\prime}\right)\right\rangle_{s^{\prime \prime}},
\end{aligned}
$$

where we have omitted for brevity all variables except the Fermi-momentum. Here, $\hat{u}\left(s, s^{\prime}\right)=u\left(s, s^{\prime}\right) \hat{1}$ is the impurity scattering potential, and $\langle\ldots\rangle_{s^{\prime}}$ denotes a Fermi surface average over $s^{\prime}$.

We expand $\hat{g}$ as in Eq. E5). The zeroth order term for the Green function is given analogously to the discussion for the diffusive limit by

$$
\hat{g}^{(0)}=(-i \pi) \operatorname{sgn}\left(\varepsilon_{n}\right) \hat{\tau}_{3} .
$$

For the higher orders we need to expand the impurity $t$-matrix in the parameter $\left(T_{c 0} / \varepsilon_{n}\right)$,

$$
\hat{t}=\hat{t}^{(0)}+\hat{t}^{(1)}+\hat{t}^{(2)}+\ldots,
$$

and similarly for the impurity self energy. Introducing the operator

$$
\hat{D}\left(s, s^{\prime}\right)=\delta\left(s-s^{\prime}\right) \hat{1}-u\left(s, s^{\prime}\right) \mathcal{N}_{F}\left(s^{\prime}\right) \hat{g}^{(0)}
$$

the $t$-matrix equation for the zeroth order term $\hat{t}^{(0)}$ takes the form

$$
\left\langle\hat{D}\left(s, s^{\prime \prime}\right) \hat{t}^{(0)}\left(s^{\prime \prime}, s^{\prime}\right)\right\rangle_{s^{\prime \prime}}=\hat{u}\left(s, s^{\prime}\right) .
$$

With the inverse operator $\hat{D}^{-1}$ defined by

$$
\left\langle\hat{D}^{-1}\left(s, s^{\prime \prime}\right) \hat{D}\left(s^{\prime \prime}, s^{\prime}\right)\right\rangle_{s^{\prime \prime}}=\delta\left(s-s^{\prime}\right) \hat{1}
$$

the formal solutions are given by

$$
\begin{aligned}
& \hat{t}^{(0)}\left(s, s^{\prime}\right)=\left\langle\hat{D}^{-1}\left(s, s^{\prime \prime}\right) \hat{u}\left(s^{\prime \prime}, s^{\prime}\right)\right\rangle_{s^{\prime \prime}} \\
& \hat{t}^{(1)}\left(s, s^{\prime}\right)=\left\langle\hat{t}^{(0)}\left(s, s^{\prime \prime}\right) \mathcal{N}_{F}\left(s^{\prime \prime}\right) \hat{g}^{(1)}\left(s^{\prime \prime}\right) \hat{t}^{(0)}\left(s^{\prime \prime}, s^{\prime}\right)\right\rangle_{s^{\prime \prime}} .
\end{aligned}
$$


From Eq. (E26) we obtain,

$$
\left[\hat{\sigma}_{i m p}^{(0)}, \hat{g}^{(0)}\right]=\hat{0}
$$

as a result of $\left[\hat{u}, \hat{\tau}_{3}\right]=\hat{0}$. Consequently, the first order term for $\hat{g}$ is, in complete analogy to the discussion leading to Eq. (E12), given by

$$
\hat{g}^{(1)}=\frac{\pi}{\left|\varepsilon_{n}\right|} \hat{\Delta}
$$

Finally, for the second order term $\hat{g}^{(2)}$, we have

$$
\left[i \varepsilon_{n} \hat{\tau}_{3}, \hat{g}^{(2)}\right]=\left[\hat{\sigma}_{i m p}^{(1)}, \hat{g}^{(0)}\right]+\left[\hat{\sigma}_{i m p}^{(0)}, \hat{g}^{(1)}\right]-i \vec{v}_{F} \cdot \nabla \hat{g}^{(1)}
$$

We solve this equation by using the normalization condition, Eq. E14 . Restricting ourselves to isotropic impurity scattering, we obtain 56

$$
\begin{aligned}
& \hat{g}^{(2)}=\frac{(-i \pi)}{2 \varepsilon_{n}\left|\varepsilon_{n}\right|} \hat{\tau}_{3} \quad\left(i \vec{v}_{F} \cdot \nabla \hat{\Delta}-\hat{\Delta}^{2}+\right. \\
& \left.\frac{i \operatorname{sgn}\left(\varepsilon_{n}\right)}{\tau} \hat{\tau}_{3}\left\{\hat{\Delta}-\langle\hat{\Delta}\rangle_{s}\right\}\right),
\end{aligned}
$$

where the inverse scattering time is defined as,

$$
\frac{1}{\tau}=2 \pi c \mathcal{N}_{F} \frac{u^{2}}{1+\pi^{2} \mathcal{N}_{F}^{2} u^{2}} .
$$

For an isotropic ( $s$-wave) superconducting order parameter the last term in Eq. E30 vanishes. In this case, the second order high-energy contribution from Eq. (E30) is odd in frequency, and it drops out of the Matsubara sum. The leading order contribution comes in third order $^{56}$ and the high-energy tail of the Matsubara sum is $\propto 1 /\left|\varepsilon_{n}\right|^{3}$. This means that the technical cut-off $\varepsilon_{c}$ can be chosen much smaller than in the diffusive limit within the Usadel approximation.

The different high-energy asymptotics within the Eilenberger and Usadel approaches are due to the diffusive approximation employed by Usadel: the impurity self-energy, i.e. the inverse scattering time $1 / \tau$, is at the outset assumed to be the largest energy scale in the problem. The high-energy tail is different depending on the order in which the limits $\tau \rightarrow 0$ and $\varepsilon_{c} \rightarrow \infty$ are taken.

\section{APPENDIX F: ANALYTIC SUMMATION OF THE HIGH-ENERGY TAIL IN THE FOURIER SERIES APPROACH}

At high energies $\varepsilon_{n} \gg T_{c 0}$ and $J$, the matrix $\hat{W}$ has a simple energy dependence that we exploit to sum the
Matsubara sum to infinity. That is, we write

$$
m_{l p}=\bar{m}_{l p}+\mathcal{R}_{l p}
$$

where $\bar{m}_{l p}$ includes terms in the sum in Eqs. (D3)-(D4) up to a technical cut-off $\varepsilon_{c}$ while the rest term $\mathcal{R}_{l p}$ is the sum from $\varepsilon_{c}$ to infinity computed analytically below.

At high energies $W_{12}=-W_{21} \approx 0$, while

$$
\begin{aligned}
& W_{11} \approx \frac{\gamma_{1}}{1+\gamma_{b 1} \lambda}, \\
& W_{22} \approx-\frac{\gamma_{2}}{1+\gamma_{b 2} \lambda}
\end{aligned}
$$

where $\lambda^{2}=\varepsilon_{n} / \pi T_{c 0}$. These relations hold for both the trilayer and the pentalayer, which reflects the fact that the theory becomes local at high energies (see the effective boundary condition (6) ). The key function of the Fourier method then has the form

$$
b_{l p}=\frac{\xi_{S}}{d_{S}} \frac{2}{\lambda} \frac{c_{1}+c_{2} \lambda}{c_{3}+c_{4} \lambda+c_{5} \lambda^{2}},
$$

where

$$
\begin{aligned}
& c_{1}=\gamma_{1}+(-1)^{l+p} \gamma_{2}+\gamma_{1} \gamma_{2}\left[1+(-1)^{l+p}\right] \\
& c_{2}=\gamma_{1} \gamma_{b 2}+(-1)^{l+p} \gamma_{2} \gamma_{b 1} \\
& c_{3}=1+\gamma_{1}+\gamma_{2}+\gamma_{1} \gamma_{2} \\
& c_{4}=\gamma_{1} \gamma_{b 2}+\gamma_{2} \gamma_{b 1}+\gamma_{b 1}+\gamma_{b 2} \\
& c_{5}=\gamma_{b 1} \gamma_{b 2}
\end{aligned}
$$

For each element of the matrix $m_{l p}$ we can perform the high-energy Matsubara sum by integration. We get

$$
\begin{aligned}
\mathcal{R}_{l p} & =\delta_{l p} \frac{1}{\pi} \ln \left(1+\frac{p^{2}}{\tilde{d}_{s}^{2}} \frac{T_{c 0}}{\varepsilon_{c}}\right)+\frac{2}{\pi^{2}} \frac{1}{\tilde{d}_{S}} I_{l p}, \\
I_{l p} & =\int_{\frac{\varepsilon_{c}}{T_{c 0}}}^{\infty} \frac{c_{1} \sqrt{x}+c_{2} x}{\left(x+\frac{l^{2}}{\tilde{d}_{s}^{2}}\right)\left(x+\frac{p^{2}}{\tilde{d}_{s}^{2}}\right)\left(c_{3}+c_{4} \sqrt{x}+c_{5} x\right)} d x
\end{aligned}
$$

where we used the short hand notation $\tilde{d}_{s}=d_{s} / \pi \xi_{S}$. Note that Eq. [F10 is independent of the temperature $T$ and only depends on the parameters in Eqs. (F5)- (F9), on $d_{s}$ and on the cut-off $\varepsilon_{c}$.
1 A.I. Buzdin and M. Yu. Kupriyanov, JETP Lett. 52, 487 (1990).
2 Z. Radovic, M. Ledvij, L. Dobrosavljevic-Grujic, A.I. Buzdin, and J.R. Clem, Phys. Rev. B 44, 759 (1991). 
3 A.I. Buzdin, B. Bujicic, and M.Yu. Kupriyanov, Sov. Phys. JETP 74, 124 (1992).

4 E.A. Demler, G.B. Arnold, and M.R. Beasley, Phys. Rev. B 55, 15174 (1997).

${ }^{5}$ L.R. Tagirov, Phys. Rev. Lett. 83, 2058 (1999).

${ }^{6}$ A.I. Buzdin, A.V. Vedyayev, and N.V. Ryzhanova, Europhys. Lett. 48, 686 (1999).

7 I. Baladié, A. Buzdin, N. Ryzhanova, and A. Vedyayev, Phys. Rev. B 63, 054518 (2001).

8 Ya.V. Fominov, N.M. Chtchelkatchev, and A.A. Golubov, JEPT Lett. 74, 96 (2001); Phys. Rev. B 66, 014507 (2002).

${ }^{9}$ I. Baladié and A. Buzdin, Phys. Rev. B 67, 014523 (2003).

10 Y.V. Fominov, A.A. Golubov, and M.Y. Kupriyanov, JETP Letters 77, 510 (2003).

11 C.-Y. You, Ya.B. Bazaliy, J.Y. Gu, S.-J. Oh, L.M. Litvak, and S.D. Bader, Phys. Rev. B 70, 014505 (2004).

12 J.S. Jiang, D. Davidović, D. H. Reich, and C.L. Chien, Phys. Rev. Lett. 74, 314 (1995).

13 Th. Mühge, N.N. Garif'yanov, Yu. V. Goryunov, G.G. Khaliullin, L.R. Tagirov, K. Westerholt, I.A. Garifullin, and H. Zabel, Phys. Rev. Lett. 77, 1857 (1996).

14 J. Aarts, J.M.E. Geers, E. Brück, A.A. Golubov, and R. Coehoorn, Phys. Rev. B 56, 2779 (1997).

${ }^{15}$ L. Lazar, K. Westerholt, H. Zabel, L.R. Tagirov, Yu. V. Goryunov, N.N. Garif'yanov, and I.A. Garifullin, Phys. Rev. B 61, 3711 (2000).

16 J.Y. Gu, C.-Y. You, J.S. Jiang, J. Pearson, Ya. B. Bazaliy, and S.D. Bader, Phys. Rev. Lett. 89, 267001 (2002).

17 Y. Obi, M. Ikebe, and H. Fujishiro, Phys. Rev. Lett. 94, 057008 (2005).

18 C. Cirillo, S.L. Prischepa, M. Salvato, C. Attanasio, M. Hesselberth, and J. Aarts, Phys. Rev. B 72, 144511 (2005).

19 A. Potenza and C. H. Marrows, Phys. Rev. B 71, 180503(R) (2005).

20 I.C. Moraru, W.P. Pratt, and N.O. Birge, Phys. Rev. Lett. 96, 037004 (2006).

21 A. Yu. Rusanov, S. Habraken, and J. Aarts, Phys. Rev. B 73, 060505(R) (2006).

22 A.Yu. Rusanov, M. Hesselberth, J. Aarts, and A.I. Buzdin, Phys. Rev. Lett. 93, 057002 (2004).

23 A.I. Buzdin, Rev. Mod. Phys. 77, 935 (2005).

24 A.I. Buzdin, L.N. Bulaevskii, and S.V. Panyukov, JETP Lett. 35, 178 (1982).

25 A.I. Buzdin and M. Yu. Kupriyanov, JETP Lett. 53, 321 (1991)

26 V.V. Ryazanov, V.A. Oboznov, A. Yu. Rusanov, A.V. Veretennikov, A.A. Golubov, and J. Aarts, Phys. Rev. Lett. 86, 2427 (2001)

${ }^{27}$ H. Sellier, C. Baraduc, F. Lefloch, and R. Calemczuk, Phys. Rev. Lett. 92, 257005 (2004).

28 S.M. Frolov, D.J. Van Harlingen, V.A. Oboznov, V.V. Bolginov, and V.V. Ryazanov, Phys. Rev. B 70, 144505 (2004).

29 T. Kontos, M. Aprili, J. Lesueur, F. Genêt, B. Stephanidis, and R. Boursier, Phys. Rev. Lett. 89, 137007 (2002).

30 Y. Blum, A. Tsukernik, M. Karpovski, and A. Palevski, Phys. Rev. Lett. 89, 187004 (2002).

31 W. Guichard, M. Aprili, O. Bourgeois, T. Kontos, J. Lesueur, and P. Gandit, Phys. Rev. Lett. 90, 167001 (2003).

32 V. Shelukin, A. Tsukernik, M. Karpovski, Y. Blum, K.B. Efetov, A.F. Volkov, T. Champel, M. Eschrig, T. Löfwander, G. Schön, and A. Palevski, cond-mat/0512593 accepted for publication in Phys. Rev. B (2006).

33 F.S. Bergeret. A.F. Volkov, and K.B. Efetov, Phys. Rev. Lett. 86, 3140 (2001).

34 A.A. Golubov, M. Yu. Kupriyanov, and Ya. V. Fominov, JETP Lett. 75, 190 (2002).

35 Ya. M. Blanter and F.W.J. Hekking, Phys. Rev. B 69, 024525 (2004).

36 F.S. Bergeret, A.F. Volkov, and K.B. Efetov, Phys. Rev. B 64, 134506 (2001).

37 F.S Bergeret, A.F. Volkov, and K.B. Efetov, Phys. Rev. B 68, 064513 (2003).

38 F.S. Bergeret, A.F. Volkov, and K.B. Efetov, Phys. Rev. Lett. 86, 4096 (2001).

39 A.F. Volkov, F.S. Bergeret, and K.B. Efetov, Phys. Rev. Lett. 90, 117006 (2003).

40 A. Kadigrobov, R.I. Shekhter, and M. Jonson, Europhys. Lett. 54, 394 (2001) ; Low Temp. Phys. 27, 760 (2001).

41 M. Eschrig, J. Kopu, J.C. Cuevas, and G. Schön, Phys. Rev. Lett. 90, 137003 (2003).

42 T. Champel and M. Eschrig, Phys. Rev. B 71, 220506(R) (2005).

43 T. Champel and M. Eschrig, Phys. Rev. B 72, 054523 (2005).

44 T. Löfwander, T. Champel, J. Durst, and M. Eschrig, Phys. Rev. Lett. 95, 187003 (2005).

45 F.S. Bergeret, A.F. Volkov, and K.B. Efetov, Rev. Mod. Phys. 1321 (2005).

46 K.D. Usadel, Phys. Rev. Lett. 25, 507 (1970).

47 M.Yu. Kupriyanov and V.F. Lukichev, Sov. Phys. JETP 67, 1163 (1988)

48 P. Fulde and R.A. Ferrell, Phys. Rev. 135, A550 (1964).

49 A.J. Larkin and Y.N. Ovchinnikov, Zh. Eksp. Teor. Fiz. 47, 1136 (1964) [Sov. Phys. JETP 20, 762 (1965)].

50 A.B. Vorontsov and J.A. Sauls, Phys Rev B, 68, 064508 (2003).

51 A.B. Vorontsov and J.A. Sauls, cond-mat/0601565

52 J.A.X Alexander, T.P. Orlando, D. Rainer, and P.M. Tedrow, Phys. Rev. B 31, 5811 (1985).

${ }^{53}$ D. Xu, S.-K. Yip, and J.A. Sauls, Phys. Rev. B 51, 16233 (1995).

54 G. Eilenberger, Z. Phys. 214, 195 (1968).

55 A.I. Larkin and Y.N. Ovchinnikov, Zh. Eksp. Teor. Fiz. 55, 2262 (1968) [Sov. Phys. JETP 28, 1200 (1969)].

56 M. Eschrig, Ph.D. Thesis, Bayreuth University (1997). 